\title{
Stability of general virus dynamics models with both cellular and viral infections
}

\author{
A. M. Elaiw ${ }^{a, c, *}$, A. A. Raezah ${ }^{a, b}$, A. M. Shehata ${ }^{c}$ \\ ${ }^{a}$ Department of Mathematics, Faculty of Science, King Abdulaziz University, P. O. Box 80203, Jeddah 21589, Saudi Arabia. \\ ${ }^{b}$ Department of Mathematics, Faculty of Science, King Khalid University, Abha, Saudi Arabia. \\ ${ }^{c}$ Department of Mathematics, Faculty of Science, Al-Azhar University (Assiut Branch), Assiut, Egypt.
}

Communicated by Y. Hu

\begin{abstract}
We consider two general models for the virus dynamics with virus-to-target and infected-to-target infections. We assume that the virus-target and infected-target incidences, the production and clearance rates of all compartments are modeled by general nonlinear functions which satisfy a set of reasonable conditions. We incorporate the latently infected cells in the second model. For each model we prove the existence of the equilibria and calculate the basic reproduction number $\mathcal{R}_{0}$. We use suitable Lyapunov functions and apply LaSalle's invariance principle to prove the global asymptotic stability of the all equilibria of the models. We confirm the theoretical results by numerical simulations. (c) 2017 All rights reserved.
\end{abstract}

Keywords: Global stability, viral infection, cell-to-cell transfer, Lyapunov function.

2010 MSC: 34D23, 37B25, 92C42.

\section{Introduction}

Mathematical models have become one of important helpful tools in understanding the dynamical behavior of many human viruses such as HIV, HTLV-I, HCV and HBV (see e.g. [1, 2, 6-13, 17-19, 22, 23, $25,27-35,37,39,42]$. The basic virus dynamics model has been given in [30] as:

$$
\begin{aligned}
\dot{\mathrm{T}} & =\rho-\mathrm{dT}-\beta \mathrm{TV}, \\
\dot{\mathrm{T}}^{*} & =\beta \mathrm{TV}-\mu \mathrm{T}^{*}, \\
\dot{\mathrm{V}} & =\mathrm{bT}^{*}-\mathrm{cV},
\end{aligned}
$$

where, $T, T^{*}$ and $V$ are the concentrations of the uninfected cells, infected cells, and free virus particles, respectively. The uninfected cells are replenished at rate $\rho$, die at rate $\mathrm{dT}$ and become infected at rate $\beta T V$, where $\beta$ is the virus-target incidence rate constant. The infected cells are die at rate $\mu T^{*}$. The virus particles are produced at rate $b T^{*}$ and cleared at rate $c V$. Parameters $\rho, d, \beta, \mu, b$ and $c$ are all

\footnotetext{
*Corresponding author

Email addresses: a_m_elaiw@yahoo.com (A. M. Elaiw), ahraizahah@gmail.com (A. A. Raezah)
} 
positive. In this model, the incidence rate is assumed to be bilinear. Moreover, the production rate of viruses and the death rate of the uninfected cells, infected cells and free virus particles are given by linear functions. The basic model may not describe the nonlinear virus dynamics during the infection stages [18]. Therefore, several works have been done to modify the basic model by considering different factors such as: immune response, [10,33], nonlinear forms of the incidence rate $[1,12,17,18,34,39]$, nonlinear production/removal rate of compartments $[10,15,18]$, latently infected cells $[2,6]$, intracellular time delay $[8,9,19,22,23,28,33]$. Georgescu and Hsieh [15] have generalized the above model by including the latent infected cells and representing the incidence rate, the production and death rate of all compartments by general nonlinear functions as:

$$
\begin{aligned}
\dot{\mathrm{T}} & =f(T)-h(V) g(T), \\
\dot{\mathrm{C}}^{*} & =h(V) g(T)-(\rho+\sigma) \psi\left(C^{*}\right), \\
\dot{\mathrm{T}}^{*} & =\sigma \psi\left(\mathrm{C}^{*}\right)-\mu \xi\left(\mathrm{T}^{*}\right), \\
\dot{\mathrm{V}} & =\mathrm{b} \xi\left(\mathrm{T}^{*}\right)-\mathrm{c} \varphi(\mathrm{V}),
\end{aligned}
$$

where, $\mathrm{C}(\mathrm{t})$ is the concentration of the latently infected cells. (1.1) describes the population dynamics of the latently infected cells and shows that they die at rate $\rho \psi\left(C^{*}\right)$ and they are converted to productively infected cells at rate $\sigma \psi\left(C^{*}\right)$, where $\rho$ and $\sigma$ are positive constants. $f, g, h, \psi, \xi$ and $\varphi$ are general nonlinear functions.

All the above mentioned works assume that the uninfected cells becomes infected due to virus contacts. Recently, it has been reported that the uninfected cells can also become infected due to direct contact with infected cells (see [3, 4, 14, 16, 20, 21, 24, 26, 36, 38, 40, 41]). The virus dynamics models with virusto-cell and cell-to-cell transmissions presented in $[3,4,14,16,20,21,24,26,36,38,40,41]$, assume bilinear form for the virus-target and infected-target incidences which are based on the mass action principle. Moreover, the production and death rates of the uninfected cells, infected cells and viruses are modeled by linear functions.

The aim of this paper is propose and analyze two general nonlinear virus dynamics models with both virus-to-cell and cell-to-cell infections. The virus-target and infected-target incidences, the production and clearance rates of all compartments are given by general nonlinear functions. The second model incorporates the latently infected cells as the fourth compartment. For both models we derive basic reproduction number and establish a set of conditions which are sufficient for the existence and global stability of the two equilibria of the models.

\section{Mathematical model}

We consider a general virus dynamics model with both cellular and viral infections as:

$$
\begin{aligned}
\dot{\mathrm{T}} & =f(T)-\left[h_{1}(V)+h_{2}\left(T^{*}\right)\right] g(T), \\
\dot{T}^{*} & =\left[h_{1}(V)+h_{2}\left(T^{*}\right)\right] g(T)-\mu \xi\left(T^{*}\right), \\
\dot{V} & =b \xi\left(T^{*}\right)-c \varphi(V),
\end{aligned}
$$

where $f, g, h_{1}, h_{2}, \xi$ and $\varphi$ are continuously differentiable functions and satisfy the following conditions:

Assumption 2.1 (A1).

(i) there exists $T_{0}>0$ such that $f\left(T_{0}\right)=0$ and $f(T)>0$ for $T \in\left[0, T_{0}\right)$;

(ii) $\mathrm{f}^{\prime}(\mathrm{T})<0$ for all $\mathrm{T}>0$;

(iii) there exist $s>0$ and $\bar{s}>0$ such that $f(T) \leqslant s-\bar{s} T$ for all $T \geqslant 0$.

Assumption 2.2 (A2).

(i) $h_{i}(u), \xi(u), \varphi(u), g(u)>0$ for all $u>0$ and $h_{i}(0)=\xi(0)=\varphi(0)=g(0)=0, i=1,2$; 
(ii) $h_{\mathfrak{i}}^{\prime}(u)>0, \xi^{\prime}(u)>0$ and $\varphi^{\prime}(u)>0$ for all $u \geqslant 0, i=1,2, g^{\prime}(u)>0$ for all $u>0$;

(iii) there are $c_{1}$ and $c_{2}>0$ such that $\xi(u) \geqslant c_{1} u$ and $\varphi(u) \geqslant c_{2} u$ for all $u \geqslant 0$.

Assumption $2.3(\mathrm{~A} 3)$. $\left(\frac{h_{1}(V)}{\varphi(V)}\right)^{\prime} \leqslant 0$, for all $V>0$ and $\left(\frac{h_{2}\left(T^{*}\right)}{\xi\left(T^{*}\right)}\right)^{\prime} \leqslant 0$, for all $T^{*}>0$.

\subsection{Basic properties}

\subsubsection{Properties of solutions}

The non-negativity and boundedness of the solutions of system (2.1), (2.2), (2.3) are established in the following lemma:

Lemma 2.4. Suppose that $A 1$ and $A 2$ are valid. Then there exist $n_{i}>0, i=1,2$, such that the following set is positively invariant:

$$
\Theta=\left\{\left(T, T^{*}, V\right) \in \mathbb{R}_{\geqslant 0}^{3}: 0 \leqslant T, T^{*} \leqslant n_{1}, 0 \leqslant V \leqslant n_{2}\right\}
$$

Proof. Since

$$
\begin{aligned}
\left.\dot{\mathrm{T}}\right|_{\mathrm{T}=0} & =f(0)>0, \\
\left.\dot{\mathrm{T}}^{*}\right|_{\mathrm{T}^{*}=0} & =\mathrm{h}_{1}(\mathrm{~V}) \mathrm{g}(\mathrm{T}) \geqslant 0, \quad \forall \mathrm{T}, \mathrm{V} \geqslant 0, \\
\left.\dot{\mathrm{V}}\right|_{\mathrm{V}=0} & =\mathrm{b} \xi\left(\mathrm{T}^{*}\right) \geqslant 0, \quad \forall \mathrm{T}^{*} \geqslant 0,
\end{aligned}
$$

then, $\mathbb{R}_{\geqslant 0}^{3}=\{(x, y, z) \in \mathbb{R}, x \geqslant 0, y \geqslant 0, z \geqslant 0\}$ is positively invariant for system (2.1)-(2.3).

Let $\mathrm{F}_{1}(\mathrm{t})=\mathrm{T}(\mathrm{t})+\mathrm{T}^{*}(\mathrm{t})+\frac{\mu}{2 \mathrm{~b}} \mathrm{~V}(\mathrm{t})$, then

$$
\begin{aligned}
\dot{\mathrm{F}}_{1}(t) & =f(T)-\frac{\mu}{2} \xi\left(T^{*}\right)-\frac{\mu c}{2 b} \varphi(V) \leqslant s-\bar{s} T-\frac{\mu}{2} c_{1} T^{*}-\frac{\mu c}{2 b} c_{2} V \\
& \leqslant s-\sigma_{1}\left(T+T^{*}+\frac{\mu}{2 b} V\right)=s-\sigma_{1} F_{1}(t)
\end{aligned}
$$

where $\sigma_{1}=\min \left\{\bar{s}, \frac{\mu}{2} c_{1}, c_{2}\right\}$. Then

$$
F_{1}(t) \leqslant e^{-\sigma_{1} t}\left(F_{1}(0)-\frac{s}{\sigma_{1}}\right)+\frac{s}{\sigma_{1}}
$$

Hence, $0 \leqslant F_{1}(t) \leqslant n_{1}$, if $F_{1}(0) \leqslant n_{1}$ for $t \geqslant 0$, where $n_{1}=\frac{s}{\sigma_{1}}$. It follows that, $0 \leqslant T(t), T^{*}(t) \leqslant n_{1}, 0 \leqslant$ $V(t) \leqslant n_{2}$, for all $t \geqslant 0$, if $T(0)+T^{*}(0)+\frac{\mu}{2 b} V(0) \leqslant n_{1}$, where $n_{2}=\frac{2 b}{\mu} n_{1}$. Therefore, $T(t), T^{*}(t)$ and $V(t)$ are all bounded.

\subsubsection{The equilibria and basic reproduction number}

The existence of the equilibria of the model (2.1)-(2.3) will be shown in the next lemma. Let the interior of the set $\Theta$ be denoted by $\stackrel{\circ}{\Theta}$.

Lemma 2.5. Suppose that $A 1$ and $A 2$ are valid, then

(i) if $\mathcal{R}_{0} \leqslant 1$, then there exists a single equilibrium $\mathrm{P}_{0} \in \Theta$; and

(ii) if $1<\mathcal{R}_{0}$, then there exist two equilibria $\mathrm{P}_{0} \in \Theta$ and $\mathrm{P}_{1} \in \stackrel{\circ}{\Theta}$,

where $\mathcal{R}_{0}$ is the basic reproduction number.

Proof. Let the R.H.S of system (2.1)-(2.3) be equal zero

$$
\begin{aligned}
& 0=f(T)-\left[h_{1}(V)+h_{2}\left(T^{*}\right)\right] g(T), \\
& 0=\left[h_{1}(V)+h_{2}\left(T^{*}\right)\right] g(T)-\mu \xi\left(T^{*}\right), \\
& 0=b \xi\left(T^{*}\right)-c \varphi(V) .
\end{aligned}
$$


From (2.6), $\varphi(\mathrm{V})=\frac{\mathrm{b}}{\mathrm{c}} \xi\left(\mathrm{T}^{*}\right)$ and from (2.4) and (2.5) we have

$$
\xi\left(T^{*}\right)=\frac{g(T) h_{1}(V)+g(T) h_{2}\left(T^{*}\right)}{\mu}=\frac{f(T)}{\mu} .
$$

Thus

$$
\varphi(V)=\frac{b}{\mu c} f(T) .
$$

From Assumption A2 we have $\varphi^{-1}, \xi^{-1}$ exist, continuous and strictly increasing. Therefore,

$$
\mathrm{V}=\varphi^{-1}\left(\frac{\mathrm{b}}{\mu \mathrm{c}} \mathrm{f}(\mathrm{T})\right) \text { and } \mathrm{T}^{*}=\xi^{-1}\left(\frac{\mathrm{f}(\mathrm{T})}{\mu}\right) .
$$

Substituting (2.9) into (2.4), we get

$$
g(T) h_{1}\left(\varphi^{-1}\left(\frac{b}{\mu c} f(T)\right)\right)+g(T) h_{2}\left(\xi^{-1}\left(\frac{1}{\mu} f(T)\right)\right)-f(T)=0 .
$$

Let

$$
H(T)=\left[h_{1}\left(\varphi^{-1}\left(\frac{b}{\mu c} f(T)\right)\right)+h_{2}\left(\xi^{-1}\left(\frac{f(T)}{\mu}\right)\right)\right] g(T)-f(T)=0 .
$$

Obviously from Assumptions A1 and A2, $\mathrm{H}(0)=-\mathrm{f}(0)<0$ and $\mathrm{H}\left(\mathrm{T}_{0}\right)=0$.

We note that, if $T=T_{0}$, then $T^{*}=V=0$, which gives the infection-free equilibrium $P_{0}=\left(T_{0}, 0,0\right)$. Now from (2.10), we get

$$
H^{\prime}(T)=\left[h_{1}(V)+h_{2}\left(T^{*}\right)\right] g^{\prime}(T)+g(T)\left(h_{1}^{\prime}(V) \frac{\partial V}{\partial T}+h_{2}^{\prime}\left(T^{*}\right) \frac{\partial T^{*}}{\partial T}\right)-f^{\prime}(T) .
$$

Moreover, from (2.7) and (2.8), we have

$$
\frac{\partial T^{*}}{\partial T}=\frac{f^{\prime}(T)}{\mu \xi^{\prime}\left(T^{*}\right)} \quad \text { and } \quad \frac{\partial V}{\partial T}=\frac{b f^{\prime}(T)}{\mu c \varphi^{\prime}(V)} .
$$

Substituting (2.12) into (2.11), we get

$$
H^{\prime}\left(T_{0}\right)=g^{\prime}\left(T_{0}\right)\left[h_{1}(0)+h_{2}(0)\right]+g\left(T_{0}\right)\left(h_{1}^{\prime}(0) \frac{b f^{\prime}\left(T_{0}\right)}{\mu c \varphi^{\prime}(0)}+h_{2}^{\prime}(0) \frac{f^{\prime}\left(T_{0}\right)}{\mu \xi^{\prime}(0)}\right)-f^{\prime}\left(T_{0}\right) .
$$

Assumption A2 implies that

$$
H^{\prime}\left(T_{0}\right)=f^{\prime}\left(T_{0}\right)\left(h_{1}^{\prime}(0) \frac{b g\left(T_{0}\right)}{\mu c \varphi^{\prime}(0)}+h_{2}^{\prime}(0) \frac{g\left(T_{0}\right)}{\mu \xi^{\prime}(0)}-1\right) .
$$

From Assumption A1, we have $f^{\prime}\left(T_{0}\right)<0$. Therefore, if $h_{1}^{\prime}(0) \frac{b g\left(T_{0}\right)}{\mu \mathrm{c} \varphi^{\prime}(0)}+h_{2}^{\prime}(0) \frac{\left.g\left(T_{0}\right)\right)}{\mu \xi^{\prime}(0)}>1$, then $H^{\prime}\left(T_{0}\right)<0$ and there exists $T_{1} \in\left(0, T_{0}\right)$ such that $H\left(T_{1}\right)=0$. From (2.9) and Assumptions A1 and A2, we have $V_{1}=\varphi^{-1}\left(\frac{b}{\mu \mathrm{c}} f\left(T_{1}\right)\right)>0$ and $T_{1}^{*}=\xi^{-1}\left(\frac{f\left(T_{1}\right)}{\mu}\right)>0$. It follows that, a chronic-infection equilibrium $P_{1}=\left(T_{1}, T_{1}^{*}, V_{1}\right)$ exists when $h_{1}^{\prime}(0) \frac{b g\left(T_{0}\right)}{\mu c \varphi^{\prime}(0)}+h_{2}^{\prime}(0) \frac{g\left(T_{0}\right)}{\mu \xi^{\prime}(0)}>1$. Let us define the basic infection reproduction number as:

$$
\mathcal{R}_{0}=\frac{g\left(T_{0}\right)}{\mu}\left(\frac{b h_{1}^{\prime}(0)}{c \varphi^{\prime}(0)}+\frac{h_{2}^{\prime}(0)}{\xi^{\prime}(0)}\right)
$$

The last part of the proof is to show that $\mathrm{P}_{0} \in \Theta$ and $\mathrm{P}_{1} \in \stackrel{\circ}{\Theta}$. From Assumption $\mathrm{A} 1$, we have

$$
0=f\left(T_{0}\right) \leqslant s-\bar{s} T_{0} \Rightarrow T_{0} \leqslant \frac{s}{\bar{s}} \leqslant \frac{s}{\sigma_{1}}=n_{1},
$$


then $P_{0} \in \Theta$. Now we have $T_{1}<T_{0}$, then from Assumption A1

$$
0=f\left(T_{0}\right)<f\left(T_{1}\right) \leqslant s-\bar{s} T_{1} .
$$

It follows that

$$
\mathrm{T}_{1}<\frac{\mathrm{s}}{\overline{\mathrm{s}}} \leqslant \mathrm{n}_{1}
$$

From (2.7) and Assumptions A1, A2, we get

$$
\begin{aligned}
\mu c_{1} T_{1}^{*} & \leqslant \mu \xi\left(T_{1}^{*}\right)=f\left(T_{1}\right)<f(0) \leqslant s \\
& \Rightarrow 0<T_{1}^{*}<\frac{s}{\mu c_{1}}<\frac{s}{\frac{\mu}{2} c_{1}} \leqslant n_{1} .
\end{aligned}
$$

Similarly, from (2.8) and Assumptions A1, A2, we have

$$
\begin{aligned}
\mathrm{cc}_{2} V_{1} & \leqslant c \varphi\left(V_{1}\right)=\frac{b}{\mu} f\left(T_{1}\right)<\frac{b}{\mu} f(0) \leqslant \frac{b}{\mu} s \\
& \Rightarrow 0<V_{1}<\frac{b s}{c_{2} \mu}<\frac{2 b s}{c c_{2} \mu} \leqslant n_{2} .
\end{aligned}
$$

Thus, $\mathrm{P}_{1} \in \stackrel{\circ}{\Theta}$.

\subsubsection{Global properties}

In the following we establish the global stability of the two equilibria of system (2.1)-(2.3) by constructing suitable Lyapunov functionals.

Theorem 2.6. Suppose that $\mathcal{R}_{0} \leqslant 1$ and Assumptions A1-A3 are valid, then $\mathrm{P}_{0}$ is globally asymptotically stable (GAS) in $\Theta$.

Proof. Construct the Lyapunov functional

$$
\mathrm{U}_{0}\left(\mathrm{~T}, \mathrm{~T}^{*}, \mathrm{~V}\right)=\mathrm{T}-\mathrm{T}_{0}-\int_{\mathrm{T}_{0}}^{\mathrm{T}} \frac{\mathrm{g}\left(\mathrm{T}_{0}\right)}{\mathrm{g}(\vartheta)} \mathrm{d} \vartheta+\mathrm{T}^{*}+\frac{\mathrm{g}\left(\mathrm{T}_{0}\right) \mathrm{h}_{1}^{\prime}(0)}{\mathrm{c} \varphi^{\prime}(0)} \mathrm{V}
$$

It is seen that, $\mathrm{U}_{0}\left(T, T^{*}, V\right)>0$ for all $T, T^{*}, V>0$, and $\mathrm{U}_{0}\left(\mathrm{~T}_{0}, 0,0\right)=0$. Calculating $\frac{\mathrm{d} \mathrm{U}_{0}}{\mathrm{dt}}$ along system (2.1)-(2.3), we obtain

$$
\begin{aligned}
\frac{d U_{0}}{d t}= & \left(1-\frac{g\left(T_{0}\right)}{g(T)}\right)\left(f(T)-g(T) h_{1}(V)-g(T) h_{2}\left(T^{*}\right)\right)+g(T) h_{1}(V) \\
& +g(T) h_{2}\left(T^{*}\right)-\mu \xi\left(T^{*}\right)+\frac{g\left(T_{0}\right) h_{1}^{\prime}(0)}{c \varphi^{\prime}(0)}\left(b \xi\left(T^{*}\right)-c \varphi(V)\right) \\
= & \left(1-\frac{g\left(T_{0}\right)}{g(T)}\right) f(T)+g\left(T_{0}\right) h_{1}(V)+g\left(T_{0}\right) h_{2}\left(T^{*}\right)+\left(b \frac{g\left(T_{0}\right) h_{1}^{\prime}(0)}{c \varphi^{\prime}(0)}-\mu\right) \xi\left(T^{*}\right)-\frac{g\left(T_{0}\right) h_{1}^{\prime}(0)}{\varphi^{\prime}(0)} \varphi(V) .
\end{aligned}
$$

Since $f\left(T_{0}\right)=0$ then we get

$$
\begin{aligned}
\frac{d U_{0}}{d t}= & \left(1-\frac{g\left(T_{0}\right)}{g(T)}\right)\left(f(T)-f\left(T_{0}\right)\right)+g\left(T_{0}\right) h_{1}(V)+g\left(T_{0}\right) h_{2}\left(T^{*}\right)+\left(\frac{b g\left(T_{0}\right) h_{1}^{\prime}(0)}{c \varphi^{\prime}(0)}-\mu\right) \xi\left(T^{*}\right) \\
& -\frac{g\left(T_{0}\right) h_{1}^{\prime}(0)}{\varphi^{\prime}(0)} \varphi(V) .
\end{aligned}
$$

From Assumption A3 we have

$$
\frac{h_{1}(V)}{\varphi(V)} \leqslant \lim _{V \rightarrow 0^{+}} \frac{h_{1}(V)}{\varphi(V)}=\frac{h_{1}^{\prime}(0)}{\varphi^{\prime}(0)} \quad \text { and } \quad \frac{h_{2}\left(T^{*}\right)}{\xi\left(T^{*}\right)} \leqslant \lim _{T^{*} \rightarrow 0^{+}} \frac{h_{2}\left(T^{*}\right)}{\xi\left(T^{*}\right)}=\frac{h_{2}^{\prime}(0)}{\xi^{\prime}(0)} .
$$


Then,

$$
\begin{aligned}
\frac{d U_{0}}{d t} & \leqslant\left(1-\frac{g\left(T_{0}\right)}{g(T)}\right)\left(f(T)-f\left(T_{0}\right)\right)+\mu\left(\frac{g\left(T_{0}\right) h_{2}^{\prime}(0)}{\mu \xi^{\prime}(0)}+\frac{b g\left(T_{0}\right) h_{1}^{\prime}(0)}{\mu c \varphi^{\prime}(0)}-1\right) \xi\left(T^{*}\right) \\
& =\left(1-\frac{g\left(T_{0}\right)}{g(T)}\right)\left(f(T)-f\left(T_{0}\right)\right)+\mu\left(\mathcal{R}_{0}-1\right) \xi\left(T^{*}\right)
\end{aligned}
$$

From Assumptions A1 and A2, we have

$$
\left(1-\frac{g\left(T_{0}\right)}{g(T)}\right)\left(f(T)-f\left(T_{0}\right)\right) \leqslant 0
$$

Therefore, if $\mathcal{R}_{0} \leqslant 1$, then $\frac{d U_{0}}{d t} \leqslant 0$ for all $T, T^{*}>0$. Let $D_{0}=\left\{\left(T, T^{*}, V\right): \frac{d U_{0}}{d t}=0\right\}$. It is clear that $P_{0}$ is the largest invariant subset of $\mathrm{D}_{0}$ and it follows from (2.14) that $\frac{\mathrm{dU}_{0}}{\mathrm{dt}}=0$ if and only if $\mathrm{T}(\mathrm{t})=\mathrm{T}_{0}$ and $T^{*}(t)=0$ for all $t$. For any element belongs to $D_{0}$ we have $T^{*}=0$. From (2.2) we have

$$
0=\dot{\mathrm{T}}^{*}=g(\mathrm{~T}) \mathrm{h}_{1}(\mathrm{~V}) \text {. }
$$

Assumption $A 2$ implies that $V=0$. Hence $\frac{\mathrm{du}_{0}}{\mathrm{dt}}=0$ if and only if $\mathrm{T}=\mathrm{T}_{0}, \mathrm{~T}^{*}=\mathrm{V}=0$. LaSalle's invariance principle implies that $P_{0}$ is GAS when $\mathcal{R}_{0} \leqslant 1$.

Remark 2.7. From Assumptions A1-A3 we have

$$
\begin{array}{r}
\left(\frac{h_{1}(V)}{\varphi(V)}-\frac{h_{1}\left(V_{1}\right)}{\varphi\left(V_{1}\right)}\right)\left(h_{1}(V)-h_{1}\left(V_{1}\right)\right) \leqslant 0, \\
\left(\frac{h_{2}\left(T^{*}\right)}{\xi\left(T^{*}\right)}-\frac{h_{2}\left(T_{1}^{*}\right)}{\xi\left(T_{1}^{*}\right)}\right)\left(h_{2}\left(T^{*}\right)-h_{2}\left(T_{1}^{*}\right)\right) \leqslant 0,
\end{array}
$$

and this leads to

$$
\begin{aligned}
& \left(\frac{h_{1}(\mathrm{~V})}{h_{1}\left(V_{1}\right)}-\frac{\varphi(V)}{\varphi\left(V_{1}\right)}\right)\left(1-\frac{h_{1}\left(V_{1}\right)}{h_{1}(V)}\right) \leqslant 0, \\
& \left(\frac{h_{2}\left(T^{*}\right)}{h_{2}\left(T_{1}^{*}\right)}-\frac{\xi\left(T^{*}\right)}{\xi\left(T_{1}^{*}\right)}\right)\left(1-\frac{h_{2}\left(T_{1}^{*}\right)}{h_{2}\left(T^{*}\right)}\right) \leqslant 0 .
\end{aligned}
$$

Theorem 2.8. Let Assumptions A1-A3 hold true and $\mathcal{R}_{0}>1$, then the chronic-infection equilibrium $\mathrm{P}_{1}$ is $G A S$ in $\stackrel{\circ}{\Theta}$.

Proof. Construct a Lyapunov functional

$$
\mathrm{U}_{1}\left(\mathrm{~T}, \mathrm{~T}^{*}, V\right)=\mathrm{T}-\mathrm{T}_{1}-\int_{\mathrm{T}_{1}}^{\mathrm{T}} \frac{\mathrm{g}\left(\mathrm{T}_{1}\right)}{\mathrm{g}(\vartheta)} \mathrm{d} \vartheta+\mathrm{T}^{*}-\mathrm{T}_{1}^{*}-\int_{\mathrm{T}_{1}^{*}}^{\mathrm{T}^{*}} \frac{\xi\left(\mathrm{T}_{1}^{*}\right)}{\xi(\vartheta)} \mathrm{d} \vartheta+\frac{g\left(\mathrm{~T}_{1}\right) \mathrm{h}_{1}\left(\mathrm{~V}_{1}\right)}{\mathrm{c} \varphi\left(\mathrm{V}_{1}\right)}\left(\mathrm{V}-\mathrm{V}_{1}-\int_{\mathrm{V}_{1}}^{\mathrm{V}} \frac{\varphi(\mathrm{V})}{\varphi(\vartheta)} \mathrm{d} \vartheta\right) .
$$

It is seen that, $U_{1}\left(T, T^{*}, V\right)>0$ for all $T, T^{*}, V>0$, and $U_{1}\left(T_{1}, T_{1}^{*}, V_{1}\right)=0$. Calculating $\frac{d U_{1}}{d t}$ along system (2.1)-(2.3), we obtain

$$
\begin{aligned}
\frac{d U_{1}}{d t}= & \left(1-\frac{g\left(T_{1}\right)}{g(T)}\right)\left(f(T)-g(T) h_{1}(V)-g(T) h_{2}\left(T^{*}\right)\right) \\
& +\left(1-\frac{\xi\left(T_{1}^{*}\right)}{\xi\left(T^{*}\right)}\right)\left(g(T) h_{1}(V)+g(T) h_{2}\left(T^{*}\right)-\mu \xi\left(T^{*}\right)\right)+\frac{g\left(T_{1}\right) h_{1}\left(V_{1}\right)}{c \varphi\left(V_{1}\right)}\left(1-\frac{\varphi\left(V_{1}\right)}{\varphi(V)}\right)\left(b \xi\left(T^{*}\right)-c \varphi(V)\right)
\end{aligned}
$$




$$
\begin{aligned}
= & \left(1-\frac{g\left(T_{1}\right)}{g(T)}\right)\left(f(T)-f\left(T_{1}\right)\right)+\left(1-\frac{g\left(T_{1}\right)}{g(T)}\right) f\left(T_{1}\right)+g\left(T_{1}\right) h_{1}(V)+g\left(T_{1}\right) h_{2}\left(T^{*}\right)-\mu \xi\left(T^{*}\right) \\
& -\frac{\xi\left(T_{1}^{*}\right) g(T) h_{1}(V)}{\xi\left(T^{*}\right)}-\frac{\xi\left(T_{1}^{*}\right) g(T) h_{2}\left(T^{*}\right)}{\xi\left(T^{*}\right)}+\mu \xi\left(T_{1}^{*}\right)+b \frac{g\left(T_{1}\right) h_{1}\left(V_{1}\right)}{c \varphi\left(V_{1}\right)} \xi\left(T^{*}\right) \\
& -\frac{g\left(T_{1}\right) h_{1}\left(V_{1}\right)}{c \varphi(V)} b \xi\left(T^{*}\right)-g\left(T_{1}\right) h_{1}\left(V_{1}\right) \frac{\varphi(V)}{\varphi\left(V_{1}\right)}+g\left(T_{1}\right) h_{1}\left(V_{1}\right) .
\end{aligned}
$$

Using the equilibrium conditions for $\mathrm{P}_{1}$ :

$$
\begin{aligned}
f\left(T_{1}\right) & =g\left(T_{1}\right) h_{1}\left(V_{1}\right)+g\left(T_{1}\right) h_{2}\left(T_{1}^{*}\right), \\
\mu \xi\left(T_{1}^{*}\right) & =g\left(T_{1}\right) h_{1}\left(V_{1}\right)+g\left(T_{1}\right) h_{2}\left(T_{1}^{*}\right), \\
b \xi\left(T_{1}^{*}\right) & =c \varphi\left(V_{1}\right),
\end{aligned}
$$

we obtain

$$
\begin{aligned}
\frac{d U_{1}}{d t}= & \left(1-\frac{g\left(T_{1}\right)}{g(T)}\right)\left(f(T)-f\left(T_{1}\right)\right)+\left(1-\frac{g\left(T_{1}\right)}{g(T)}\right)\left(g\left(T_{1}\right) h_{1}\left(V_{1}\right)+g\left(T_{1}\right) h_{2}\left(T_{1}^{*}\right)\right) \\
& +g\left(T_{1}\right) h_{1}\left(V_{1}\right)\left[\frac{h_{1}(V)}{h_{1}\left(V_{1}\right)}-\frac{\varphi(V)}{\varphi\left(V_{1}\right)}\right]+g\left(T_{1}\right) h_{2}\left(T_{1}^{*}\right)\left[\frac{h_{2}\left(T^{*}\right)}{h_{2}\left(T_{1}^{*}\right)}-\frac{\xi\left(T^{*}\right)}{\xi\left(T_{1}^{*}\right)}\right] \\
& +\left[\frac{g\left(T_{1}\right) h_{2}\left(T_{1}^{*}\right)}{\xi\left(T_{1}^{*}\right)}-\mu+\frac{b g\left(T_{1}\right) h_{1}\left(V_{1}\right)}{c \varphi\left(V_{1}\right)}\right] \xi\left(T^{*}\right)-\frac{\xi\left(T_{1}^{*}\right) g(T) h_{1}(V)}{\xi\left(T^{*}\right)}-\frac{\xi\left(T_{1}^{*}\right) g(T) h_{2}\left(T^{*}\right)}{\xi\left(T^{*}\right)} \\
& +g\left(T_{1}\right) h_{1}\left(V_{1}\right)+g\left(T_{1}\right) h_{2}\left(T_{1}^{*}\right)-\frac{b g\left(T_{1}\right) h_{1}\left(V_{1}\right)}{c \varphi(V)} \xi\left(T^{*}\right)+g\left(T_{1}\right) h_{1}\left(V_{1}\right) .
\end{aligned}
$$

Collecting terms of (2.15), we get

$$
\begin{aligned}
\frac{d U_{1}}{d t}= & \left(1-\frac{g\left(T_{1}\right)}{g(T)}\right)\left(f(T)-f\left(T_{1}\right)\right)+g\left(T_{1}\right) h_{1}\left(V_{1}\right)+g\left(T_{1}\right) h_{2}\left(T_{1}^{*}\right)-g\left(T_{1}\right) h_{1}\left(V_{1}\right) \frac{g\left(T_{1}\right)}{g(T)} \\
& -g\left(T_{1}\right) h_{2}\left(T_{1}^{*}\right) \frac{g\left(T_{1}\right)}{g(T)}+g\left(T_{1}\right) h_{1}\left(V_{1}\right)\left[\frac{h_{1}(V)}{h_{1}\left(V_{1}\right)}-\frac{\varphi(V)}{\varphi\left(V_{1}\right)}\right]+g\left(T_{1}\right) h_{2}\left(T_{1}^{*}\right)\left[\frac{h_{2}\left(T^{*}\right)}{h_{2}\left(T_{1}^{*}\right)}-\frac{\xi\left(T^{*}\right)}{\xi\left(T_{1}^{*}\right)}\right] \\
& -g\left(T_{1}\right) h_{1}\left(V_{1}\right) \frac{\xi\left(T_{1}^{*}\right) g(T) h_{1}(V)}{\xi\left(T^{*}\right) g\left(T_{1}\right) h_{1}\left(V_{1}\right)}-g\left(T_{1}\right) h_{2}\left(T_{1}^{*}\right) \frac{\xi\left(T_{1}^{*}\right) g(T) h_{2}\left(T^{*}\right)}{\xi\left(T^{*}\right) g\left(T_{1}\right) h_{2}\left(T_{1}^{*}\right)} \\
& +g\left(T_{1}\right) h_{1}\left(V_{1}\right)+g\left(T_{1}\right) h_{2}\left(T_{1}^{*}\right)-g\left(T_{1}\right) h_{1}\left(V_{1}\right) \frac{\xi\left(T^{*}\right) \varphi\left(V_{1}\right)}{\xi\left(T_{1}^{*}\right) \varphi(V)}+g\left(T_{1}\right) h_{1}\left(V_{1}\right) .
\end{aligned}
$$

Equation (2.16) can be simplified as:

$$
\begin{aligned}
\frac{d U_{1}}{d t}= & \left(1-\frac{g\left(T_{1}\right)}{g(T)}\right)\left(f(T)-f\left(T_{1}\right)\right)+g\left(T_{1}\right) h_{1}\left(V_{1}\right)\left[3-\frac{g\left(T_{1}\right)}{g(T)}-\frac{\xi\left(T_{1}^{*}\right) g(T) h_{1}(V)}{\xi\left(T^{*}\right) g\left(T_{1}\right) h_{1}\left(V_{1}\right)}-\frac{\xi\left(T^{*}\right) \varphi\left(V_{1}\right)}{\xi\left(T_{1}^{*}\right) \varphi(V)}\right] \\
& +g\left(T_{1}\right) h_{2}\left(T_{1}^{*}\right)\left[2-\frac{g\left(T_{1}\right)}{g(T)}-\frac{\xi\left(T_{1}^{*}\right) g(T) h_{2}\left(T^{*}\right)}{\xi\left(T^{*}\right) g\left(T_{1}\right) h_{2}\left(T_{1}^{*}\right)}\right]+g\left(T_{1}\right) h_{1}\left(V_{1}\right)\left[\frac{h_{1}(V)}{h_{1}\left(V_{1}\right)}-\frac{\varphi(V)}{\varphi\left(V_{1}\right)}\right] \\
& +g\left(T_{1}\right) h_{2}\left(T_{1}^{*}\right)\left[\frac{h_{2}\left(T^{*}\right)}{h_{2}\left(T_{1}^{*}\right)}-\frac{\xi\left(T^{*}\right)}{\xi\left(T_{1}^{*}\right)}\right] \\
= & \left(1-\frac{g\left(T_{1}\right)}{g(T)}\right)\left(f(T)-f\left(T_{1}\right)\right)+g\left(T_{1}\right) h_{1}\left(V_{1}\right)\left[\frac{h_{1}(V)}{h_{1}\left(V_{1}\right)}-\frac{\varphi(V)}{\varphi\left(V_{1}\right)}-1+\frac{\varphi(V) h_{1}\left(V_{1}\right)}{\varphi\left(V_{1}\right) h_{1}(V)}\right] \\
& +g\left(T_{1}\right) h_{1}\left(V_{1}\right)\left[4-\frac{g\left(T_{1}\right)}{g(T)}-\frac{\xi\left(T_{1}^{*}\right) g(T) h_{1}(V)}{\xi\left(T^{*}\right) g\left(T_{1}\right) h_{1}\left(V_{1}\right)}-\frac{\xi\left(T^{*}\right) \varphi\left(V_{1}\right)}{\xi\left(T_{1}^{*}\right) \varphi(V)}-\frac{\varphi(V) h_{1}\left(V_{1}\right)}{\varphi\left(V_{1}\right) h_{1}(V)}\right] \\
& +g\left(T_{1}\right) h_{2}\left(T_{1}^{*}\right)\left[3-\frac{g\left(T_{1}\right)}{g(T)}-\frac{\xi\left(T_{1}^{*}\right) g(T) h_{2}\left(T^{*}\right)}{\xi\left(T^{*}\right) g\left(T_{1}\right) h_{2}\left(T_{1}^{*}\right)}-\frac{\xi\left(T^{*}\right) h_{2}\left(T_{1}^{*}\right)}{\xi\left(T_{1}^{*}\right) h_{2}\left(T^{*}\right)}\right]
\end{aligned}
$$




$$
\begin{aligned}
+ & +g\left(T_{1}\right) h_{2}\left(T_{1}^{*}\right)\left[\frac{h_{2}\left(T^{*}\right)}{h_{2}\left(T_{1}^{*}\right)}-\frac{\xi\left(T^{*}\right)}{\xi\left(T_{1}^{*}\right)}-1+\frac{\xi\left(T^{*}\right) h_{2}\left(T_{1}^{*}\right)}{\xi\left(T_{1}^{*}\right) h_{2}\left(T^{*}\right)}\right] \\
= & \left(1-\frac{g\left(T_{1}\right)}{g(T)}\right)\left(f(T)-f\left(T_{1}\right)\right)+g\left(T_{1}\right) h_{1}\left(V_{1}\right)\left(\frac{h_{1}(V)}{h_{1}\left(V_{1}\right)}-\frac{\varphi(V)}{\varphi\left(V_{1}\right)}\right)\left(1-\frac{h_{1}\left(V_{1}\right)}{h_{1}(V)}\right) \\
& +g\left(T_{1}\right) h_{2}\left(T_{1}^{*}\right)\left(\frac{h_{2}\left(T^{*}\right)}{h_{2}\left(T_{1}^{*}\right)}-\frac{\xi\left(T^{*}\right)}{\xi\left(T_{1}^{*}\right)}\right)\left(1-\frac{h_{2}\left(T_{1}^{*}\right)}{h_{2}\left(T^{*}\right)}\right) \\
& +g\left(T_{1}\right) h_{1}\left(V_{1}\right)\left[4-\frac{g\left(T_{1}\right)}{g(T)}-\frac{\xi\left(T_{1}^{*}\right) g(T) h_{1}(V)}{\xi\left(T^{*}\right) g\left(T_{1}\right) h_{1}\left(V_{1}\right)}-\frac{\xi\left(T^{*}\right) \varphi\left(V_{1}\right)}{\xi\left(T_{1}^{*}\right) \varphi(V)}-\frac{\varphi(V) h_{1}\left(V_{1}\right)}{\varphi\left(V_{1}\right) h_{1}(V)}\right] \\
& +g\left(T_{1}\right) h_{2}\left(T_{1}^{*}\right)\left[3-\frac{g\left(T_{1}\right)}{g(T)}-\frac{\xi\left(T_{1}^{*}\right) g(T) h_{2}\left(T^{*}\right)}{\xi\left(T^{*}\right) g\left(T_{1}\right) h_{2}\left(T_{1}^{*}\right)}-\frac{\xi\left(T^{*}\right) h_{2}\left(T_{1}^{*}\right)}{\xi\left(T_{1}^{*}\right) h_{2}\left(T^{*}\right)}\right] .
\end{aligned}
$$

Using Assumptions A1-A3, we get that, the first three terms of (2.17) are less than or equal to zero. The relationship between geometrical and arithmetical means implies that the last two terms of (2.17) are also less than or equal to zero. It follows that, $\frac{\mathrm{dU}_{1}}{\mathrm{dt}} \leqslant 0$ for all $\mathrm{T}, \mathrm{T}^{*}, \mathrm{~V}>0$. The solutions of system (2.1)(2.3) limited to $D_{1}$, the largest invariant subset of $\left\{\left(T, T^{*}, V\right): \frac{d U_{1}}{d t}=0\right\}$. We have $\frac{d U_{1}}{d t}=0$ if and only if $T(t)=T_{1}, T^{*}(t)=T_{1}^{*}$ and $V(t)=V_{1}$. Therefore, $D_{1}=\left\{P_{1}\right\}$ and the global asymptotic stability of the chronic-infection equilibrium $\mathrm{P}_{1}$ follows from LaSalle's invariance principle.

\section{Model with latently infected cells}

In Section 2, we have assumed that all the infected cells are producer cells. In this section, we consider two types of infected cells, latently infected cells and productively infected cells. The model can be formulated as:

$$
\begin{aligned}
\dot{\mathrm{T}} & =f(T)-g(T) h_{1}(V)-g(T) h_{2}\left(T^{*}\right), \\
\dot{C}^{*} & =(1-\pi)\left(g(T) h_{1}(V)+g(T) h_{2}\left(T^{*}\right)\right)-(\rho+\sigma) \psi\left(C^{*}\right), \\
\dot{\mathrm{T}}^{*} & =\pi\left(g(T) h_{1}(V)+g(T) h_{2}\left(T^{*}\right)\right)+\sigma \psi\left(C^{*}\right)-\mu \xi\left(T^{*}\right), \\
\dot{V} & =b \xi\left(T^{*}\right)-c \varphi(V),
\end{aligned}
$$

where, $\mathrm{C}^{*}$ and $\mathrm{T}^{*}$ represent the concentrations of the latently infected and productively infected cells, respectively. The fractions $(1-\pi)$ and $\pi$ with $0<\pi<1$ are the probabilities that upon infection, an uninfected cell will become either latently infected or productively infected.

Assumption 3.1 (A4).

(i) $\psi$ is continuously differentiable, $\psi\left(C^{*}\right)>0$ for $C^{*}>0, \psi(0)=0$;

(ii) $\psi^{\prime}\left(C^{*}\right)>0$ for $C^{*}>0$; and

(iii) there is $c_{3}>0$ such that $\psi\left(C^{*}\right) \geqslant c_{3} C^{*}$ for $C^{*} \geqslant 0$.

\subsection{Basic properties}

\subsubsection{Properties of solutions}

Lemma 3.2. Suppose that Assumptions $A 1, A 2$ and $A 4$ are valid. Then there exist $\mathrm{N}_{i}>0, i=1,2$, such that the following set is positively invariant:

$$
\Theta_{\mathrm{L}}=\left\{\left(T, \mathrm{C}^{*}, \mathrm{~T}^{*}, \mathrm{~V}\right) \in \mathbb{R}_{\geqslant 0}^{4}: 0 \leqslant \mathrm{~T}, \mathrm{C}^{*}, \mathrm{~T}^{*} \leqslant \mathrm{~N}_{1}, 0 \leqslant \mathrm{~V} \leqslant \mathrm{~N}_{2}\right\}
$$

Proof. We have

$$
\begin{aligned}
\left.\dot{\mathrm{T}}\right|_{\mathrm{T}=0} & =f(0)>0, \\
\left.\dot{\mathrm{C}}^{*}\right|_{\mathrm{C}^{*}=0} & =(1-\pi)\left(g(T) h_{1}(V)+g(T) h_{2}\left(T^{*}\right)\right) \geqslant 0, \quad \forall T, T^{*}, V \geqslant 0,
\end{aligned}
$$




$$
\begin{aligned}
\left.\dot{\mathrm{T}}^{*}\right|_{\mathrm{T}^{*}=0} & =\pi \mathrm{g}(\mathrm{T}) \mathrm{h}_{1}(\mathrm{~V})+\sigma \psi\left(\mathrm{C}^{*}\right) \geqslant 0, \quad \forall \mathrm{T}, \mathrm{C}^{*}, \mathrm{~V} \geqslant 0, \\
\left.\quad \dot{\mathrm{V}}\right|_{\mathrm{V}=0} & =\mathrm{b} \xi\left(\mathrm{T}^{*}\right) \geqslant 0, \quad \forall \mathrm{T}^{*} \geqslant 0 .
\end{aligned}
$$

Hence, $\mathbb{R}_{\geqslant 0}^{4}$ is positively invariant for system (3.1), (3.2), (3.3), (3.4). Let

$$
F_{2}(t)=T(t)+C^{*}(t)+T^{*}(t)+\frac{\mu}{2 b} V(t) .
$$

Then

$$
\begin{aligned}
\dot{\mathrm{F}}_{2}(t) & =f(T)-\rho \psi\left(C^{*}\right)-\frac{\mu}{2} \xi\left(T^{*}\right)-\frac{\mu c}{2 b} \varphi(V) \leqslant s-\bar{s} T-\rho c_{3} C^{*}-\frac{\mu}{2} c_{1} T^{*}-\frac{\mu c}{2 b} c_{2} V \\
& \leqslant s-\sigma_{2}\left(T+C^{*}+T^{*}+\frac{\mu}{2 b} V\right)=s-\sigma_{2} F_{2}(t),
\end{aligned}
$$

where, $\sigma_{2}=\min \left\{\overline{\mathrm{s}}, \rho c_{3}, \frac{\mu}{2} c_{1}, \mathrm{cc}_{2}\right\}$. Then

$$
F_{2}(t) \leqslant e^{-\sigma_{2} t}\left(F_{2}(0)-\frac{s}{\sigma_{2}}\right)+\frac{s}{\sigma_{2}} .
$$

Hence, $0 \leqslant F_{2}(t) \leqslant N_{1}$ if $F_{2}(0) \leqslant N_{1}$ for $t \geqslant 0$ where $N_{1}=\frac{s}{\sigma_{2}}$. It follows that, $0 \leqslant T(t), C^{*}(t), T^{*}(t) \leqslant N_{1}$, $0 \leqslant V(t) \leqslant N_{2}$, for all $t \geqslant 0$, if $T(0)+C^{*}(0)+T^{*}(0)+\frac{\mu}{2 b} V(0) \leqslant N_{1}$, where $N_{2}=\frac{2 b s}{\mu \sigma_{2}}$. Therefore, $\mathrm{T}(\mathrm{t}), \mathrm{C}^{*}(\mathrm{t}), \mathrm{T}^{*}(\mathrm{t})$ and $\mathrm{V}(\mathrm{t})$ are all bounded.

\subsubsection{The equilibria and bifurcation parameter}

The existence of the equilibria of model (3.1)-(3.4) will be shown in the next lemma.

Lemma 3.3. Suppose that Assumptions A1, A2 and A4 are satisfied, then

(i) if $\mathcal{R}_{0}^{\mathrm{L}} \leqslant 1$, then there exists a single equilibrium $\mathrm{P}_{0} \in \Theta_{\mathrm{L}}$; and

(ii) if $1<\mathcal{R}_{0}^{\mathrm{L}}$, then there exist two positive equilibria $\mathrm{P}_{0} \in \Theta_{\mathrm{L}}$ and $\mathrm{P}_{1} \in \stackrel{\circ}{\Theta}_{\mathrm{L}}$,

where $\mathcal{R}_{0}^{\mathrm{L}}$ is the basic reproduction number.

Proof. Let the R.H.S of system (3.1)-(3.4) be equal zero

$$
\begin{aligned}
& 0=f(T)-\left[h_{1}(V)+h_{2}\left(T^{*}\right)\right] g(T), \\
& 0=(1-\pi)\left[h_{1}(V)+h_{2}\left(T^{*}\right)\right] g(T)-(\rho+\sigma) \psi\left(C^{*}\right), \\
& 0=\pi\left[h_{1}(V)+h_{2}\left(T^{*}\right)\right] g(T)+\sigma \psi\left(C^{*}\right)-\mu \xi\left(T^{*}\right), \\
& 0=b \xi\left(T^{*}\right)-c \varphi(V) .
\end{aligned}
$$

From (3.8), $\varphi(\mathrm{V})=\frac{\mathrm{b}}{\mathrm{c}} \xi\left(\mathrm{T}^{*}\right)$ and from (3.5), (3.6), (3.7) we have

$$
\psi\left(C^{*}\right)=\frac{(1-\pi) f(T)}{\rho+\sigma}, \quad \xi\left(T^{*}\right)=\frac{(\pi \rho+\sigma) f(T)}{\mu(\rho+\sigma)}, \quad \varphi(V)=\frac{b(\pi \rho+\sigma) f(T)}{c \mu(\rho+\sigma)} .
$$

It follows that

$$
C^{*}=\psi^{-1}\left(\frac{(1-\pi) f(T)}{\rho+\sigma}\right), \quad T^{*}=\xi^{-1}\left(\frac{(\pi \rho+\sigma) f(T)}{\mu(\rho+\sigma)}\right), \quad V=\varphi^{-1}\left(\frac{b(\pi \rho+\sigma) f(T)}{c \mu(\rho+\sigma)}\right) .
$$

Substituting (3.10) into (3.5) we get

$$
g(T) h_{1}\left(\varphi^{-1}\left(\frac{b(\pi \rho+\sigma) f(T)}{c \mu(\rho+\sigma)}\right)\right)+g(T) h_{2}\left(\xi^{-1}\left(\frac{(\pi \rho+\sigma) f(T)}{\mu(\rho+\sigma)}\right)\right)-f(T)=0,
$$


and let

$$
H_{L}(T)=g(T)\left[h_{1}\left(\varphi^{-1}\left(\frac{b(\rho \pi+\sigma) f(T)}{c \mu(\rho+\sigma)}\right)\right)+h_{2}\left(\xi^{-1}\left(\frac{(\pi \rho+\sigma) f(T)}{\mu(\rho+\sigma)}\right)\right)\right]-f(T)=0 .
$$

Obviously from Assumptions A1 and A2, $\mathrm{H}_{\mathrm{L}}(0)=-\mathrm{f}(0)<0$ and $\mathrm{H}_{\mathrm{L}}\left(\mathrm{T}_{0}\right)=0$.

We note that, if $T=T_{0}$ then from (3.10) we have $C^{*}=0, T^{*}=V=0$ which gives the infection-free equilibrium $P_{0}=\left(T_{0}, 0,0,0\right)$.

Now from (3.11) we get

$$
H_{L}^{\prime}(T)=g^{\prime}(T)\left[h_{1}(V)+h_{2}\left(T^{*}\right)\right]+g(T)\left(h_{1}^{\prime}(V) \frac{\partial V}{\partial T}+h_{2}^{\prime}\left(T^{*}\right) \frac{\partial T^{*}}{\partial T}\right)-f^{\prime}(T) .
$$

From (3.9) we have

$$
\frac{\partial T^{*}}{\partial T}=\frac{(\pi \rho+\sigma) f^{\prime}(T)}{\mu(\rho+\sigma) \xi^{\prime}\left(T^{*}\right)}, \quad \text { and } \quad \frac{\partial V}{\partial T}=\frac{b(\pi \rho+\sigma) f^{\prime}(T)}{c \mu(\rho+\sigma) \varphi^{\prime}(V)}
$$

Substituting (3.13) into (3.12), we get

$$
H_{L}^{\prime}\left(T_{0}\right)=g^{\prime}\left(T_{0}\right)\left[h_{1}(0)+h_{2}(0)\right]+g\left(T_{0}\right)\left(h_{1}^{\prime}(0) \frac{b(\pi \rho+\sigma) f^{\prime}\left(T_{0}\right)}{c \mu(\rho+\sigma) \varphi^{\prime}(0)}+h_{2}^{\prime}(0) \frac{(\pi \rho+\sigma) f^{\prime}\left(T_{0}\right)}{\mu(\rho+\sigma) \xi^{\prime}(0)}\right)-f^{\prime}\left(T_{0}\right) .
$$

Assumption A2 implies that $h_{1}(0), h_{2}(0)=0, g\left(T_{0}\right), h_{1}^{\prime}(0), h_{2}^{\prime}(0), \varphi^{\prime}(0)$ and $\xi^{\prime}(0)>0$ then

$$
H_{L}^{\prime}\left(T_{0}\right)=f^{\prime}\left(T_{0}\right)\left(h_{1}^{\prime}(0) \frac{b(\pi \rho+\sigma) g\left(T_{0}\right)}{c \mu(\rho+\sigma) \varphi^{\prime}(0)}+h_{2}^{\prime}(0) \frac{(\pi \rho+\sigma) g\left(T_{0}\right)}{\mu(\rho+\sigma) \xi^{\prime}(0)}-1\right) .
$$

From Assumption A1, we have $f^{\prime}\left(T_{0}\right)<0$. Therefore, if $h_{1}^{\prime}(0) \frac{b(\pi \rho+\sigma) g\left(T_{0}\right)}{c \mu(\rho+\sigma) \varphi^{\prime}(0)}+h_{2}^{\prime}(0) \frac{(\pi \rho+\sigma) g\left(T_{0}\right)}{\mu(\rho+\sigma) \xi^{\prime}(0)}>1$, then $\mathrm{H}_{\mathrm{L}}^{\prime}\left(\mathrm{T}_{0}\right)<0$ and there exists $\mathrm{T}_{1} \in\left(0, \mathrm{~T}_{0}\right)$ such that $\mathrm{H}_{\mathrm{L}}\left(\mathrm{T}_{1}\right)=0$, moreover,

$$
C_{1}^{*}=\psi^{-1}\left(\frac{(1-\pi) f\left(T_{1}\right)}{\rho+\sigma}\right)>0, \quad T_{1}^{*}=\xi^{-1}\left(\frac{(\pi \rho+\sigma) f\left(T_{1}\right)}{\mu(\rho+\sigma)}\right)>0, \quad V_{1}=\varphi^{-1}\left(\frac{b(\pi \rho+\sigma) f\left(T_{1}\right)}{c \mu(\rho+\sigma)}\right)>0 .
$$

It follows that a chronic-infection equilibrium $P_{1}=\left(T_{1}, C_{1}^{*}, T_{1}^{*}, V_{1}\right)$ exists when

$$
h_{1}^{\prime}(0) \frac{b(\pi \rho+\sigma) g\left(T_{0}\right)}{c \mu(\rho+\sigma) \varphi^{\prime}(0)}+h_{2}^{\prime}(0) \frac{(\pi \rho+\sigma) g\left(T_{0}\right)}{\mu(\rho+\sigma) \xi^{\prime}(0)}>1 .
$$

We define the basic infection reproduction number

$$
\mathcal{R}_{0}^{\mathrm{L}}=\frac{(\pi \rho+\sigma) g\left(\mathrm{~T}_{0}\right)}{\mu(\rho+\sigma)}\left(\frac{\mathrm{bh} h_{1}^{\prime}(0)}{\mathrm{c} \varphi^{\prime}(0)}+\frac{\mathrm{h}_{2}^{\prime}(0)}{\xi^{\prime}(0)}\right) .
$$

Clearly $\mathrm{P}_{0} \in \Theta_{\mathrm{L}}$. Now we show that $\mathrm{P}_{1} \in \stackrel{\circ}{\Theta}_{\mathrm{L}}$. We have $\mathrm{T}_{1}<\mathrm{T}_{0}$, then from Assumption A1

$$
\begin{aligned}
0 & =f\left(T_{0}\right)<f\left(T_{1}\right) \leqslant s-\bar{s} T_{1} \\
& \Rightarrow T_{1}<\frac{s}{\bar{s}} \leqslant N_{1} .
\end{aligned}
$$

From (3.9) and Assumptions A1, A4, we get

$$
\begin{aligned}
c_{3} C_{1}^{*} & \leqslant \psi\left(C_{1}^{*}\right)=\frac{(1-\pi) f\left(T_{1}\right)}{\rho+\sigma}<\frac{(1-\pi)}{(\rho+\sigma)} f(0) \leqslant \frac{(1-\pi)}{(\rho+\sigma)} s<\frac{(1-\pi)}{\rho} s<\frac{s}{\rho} \\
& \Rightarrow 0<C_{1}^{*}<\frac{s}{\rho c_{3}} \leqslant N_{1} .
\end{aligned}
$$


In addition, from (3.9) and Assumptions A1, A2, we have

$$
\begin{aligned}
\mu c_{1} \mathrm{~T}_{1}^{*} & \leqslant \mu \xi\left(\mathrm{T}_{1}^{*}\right)=\frac{\pi \rho+\sigma}{\rho+\sigma} \mathrm{f}\left(\mathrm{T}_{1}\right)<\frac{\pi \rho+\sigma}{\rho+\sigma} \mathrm{f}(0)<\frac{\pi \rho+\sigma}{\rho+\sigma} \mathrm{s}<\mathrm{s} \\
& \Rightarrow \mathrm{T}_{1}^{*}<\frac{\mathrm{s}}{\mu \mathrm{c}_{1}}<\frac{\mathrm{s}}{\frac{\mu}{2} \mathrm{c}_{1}} \leqslant \mathrm{~N}_{1} .
\end{aligned}
$$

Similarly, from (3.9) and Assumptions A1, A2, we have

$$
\begin{aligned}
\operatorname{cc}_{2} V_{1} & \leqslant c \varphi\left(V_{1}\right)=\frac{b(\pi \rho+\sigma) f\left(T_{1}\right)}{\mu(\rho+\sigma)}<\frac{b(\pi \rho+\sigma) f(0)}{\mu(\rho+\sigma)} \leqslant \frac{b(\pi \rho+\sigma) s}{\mu(\rho+\sigma)} \leqslant \frac{b}{\mu} s \\
& \Rightarrow 0<V_{1}<\frac{b s}{c c_{2} \mu}<\frac{2 b s}{c c_{2} \mu} \leqslant N_{2} .
\end{aligned}
$$

Thus, $\mathrm{P}_{1} \in \stackrel{\circ}{\Theta}_{\mathrm{L}}$.

\subsubsection{Global properties of the model with latently infected cells}

In the following we establish the global stability of the two equilibria of model (3.1)-(3.4) by constructing suitable Lyapunov functionals.

Theorem 3.4. Suppose that $\mathcal{R}_{0}^{\mathrm{L}} \leqslant 1$ and A1-A4 are valid, then for system (3.1)-(3.4), $\mathrm{P}_{0}$ is GAS in $\Theta_{\mathrm{L}}$.

Proof. Constructing a Lyapunov functional $\mathrm{U}_{0}^{\mathrm{L}}\left(\mathrm{T}, \mathrm{C}^{*}, \mathrm{~T}^{*}, \mathrm{~V}\right)$ as

$$
\mathrm{U}_{0}^{\mathrm{L}}\left(\mathrm{T}, \mathrm{C}^{*}, \mathrm{~T}^{*}, V\right)=\mathrm{T}-\mathrm{T}_{0}-\int_{\mathrm{T}_{0}}^{\mathrm{T}} \frac{\mathrm{g}\left(\mathrm{T}_{0}\right)}{\mathrm{g}(\vartheta)} \mathrm{d} \vartheta+\frac{\sigma}{\pi \rho+\sigma} \mathrm{C}^{*}+\frac{\rho+\sigma}{\pi \rho+\sigma} \mathrm{T}^{*}+\frac{g\left(\mathrm{~T}_{0}\right) \mathrm{h}_{1}^{\prime}(0)}{\mathrm{c} \varphi^{\prime}(0)} \mathrm{V} .
$$

Calculating $\frac{\mathrm{du}_{0}^{\mathrm{L}}}{\mathrm{dt}}$ along system (3.1)-(3.4), we obtain

$$
\begin{aligned}
\frac{d u_{0}^{L}}{d t}= & \left(1-\frac{g\left(T_{0}\right)}{g(T)}\right)\left(f(T)-g(T) h_{1}(V)-g(T) h_{2}\left(T^{*}\right)\right) \\
& \left.+\frac{\sigma}{\pi \rho+\sigma}\left((1-\pi) h_{1}(V)+h_{2}\left(T^{*}\right)\right) g(T)-(\rho+\sigma) \psi\left(C^{*}\right)\right) \\
& +\frac{\rho+\sigma}{\pi \rho+\sigma}\left(\pi\left(h_{1}(V)+h_{2}\left(T^{*}\right)\right) g(T)+\sigma \psi\left(C^{*}\right)-\mu \xi\left(T^{*}\right)\right)+\frac{g\left(T_{0}\right) h_{1}^{\prime}(0)}{c \varphi^{\prime}(0)}\left(b \xi\left(T^{*}\right)-c \varphi(V)\right) \\
= & \left(1-\frac{g\left(T_{0}\right)}{g(T)}\right) f(T)+g\left(T_{0}\right) h_{1}(V)+g\left(T_{0}\right) h_{2}\left(T^{*}\right)+\left(\frac{b g\left(T_{0}\right) h_{1}^{\prime}(0)}{c \varphi^{\prime}(0)}-\frac{\mu(\rho+\sigma)}{\pi \rho+\sigma}\right) \xi\left(T^{*}\right) \\
& -\frac{g\left(T_{0}\right) h_{1}^{\prime}(0)}{\varphi^{\prime}(0)} \varphi(V) .
\end{aligned}
$$

Since $f\left(T_{0}\right)=0$ then we get

$$
\begin{aligned}
\frac{d U_{0}^{L}}{d t}= & \left(1-\frac{g\left(T_{0}\right)}{g(T)}\right)\left(f(T)-f\left(T_{0}\right)\right)+g\left(T_{0}\right) h_{1}(V)+g\left(T_{0}\right) h_{2}\left(T^{*}\right)+\left(\frac{b g\left(T_{0}\right) h_{1}^{\prime}(0)}{c \varphi^{\prime}(0)}-\frac{\rho+\sigma}{\pi \rho+\sigma} \mu\right) \xi\left(T^{*}\right) \\
& -\frac{g\left(T_{0}\right) h_{1}^{\prime}(0)}{\varphi^{\prime}(0)} \varphi(V) .
\end{aligned}
$$

Applying (2.13), we get

$$
\begin{aligned}
\frac{d U_{0}^{L}}{d t} & \leqslant\left(1-\frac{g\left(T_{0}\right)}{g(T)}\right)\left(f(T)-f\left(T_{0}\right)\right)+\left(\frac{g\left(T_{0}\right) h_{2}^{\prime}(0)}{\xi^{\prime}(0)}+\frac{b g\left(T_{0}\right) h_{1}^{\prime}(0)}{c \varphi^{\prime}(0)}-\frac{\mu(\rho+\sigma)}{\pi \rho+\sigma}\right) \xi\left(T^{*}\right) \\
& =\left(1-\frac{g\left(T_{0}\right)}{g(T)}\right)\left(f(T)-f\left(T_{0}\right)\right)+\frac{\mu(\rho+\sigma)}{\pi \rho+\sigma}\left(\mathcal{R}_{0}^{L}-1\right) \xi\left(T^{*}\right) .
\end{aligned}
$$


Therefore, if $\mathcal{R}_{0}^{\mathrm{L}} \leqslant 1$, then $\frac{\mathrm{dU}}{\mathrm{dt}} \leqslant 0$ for all $\mathrm{T}, \mathrm{T}^{*}>0$. Similar to the previous section, one can show that $\mathrm{P}_{0}$ is GAS.

Theorem 3.5. Suppose that $\mathcal{R}_{0}^{\mathrm{L}}>1$ and $A 1-A 3$ are valid, then for system (3.1)-(3.4), $\mathrm{P}_{1}$ is GAS in $\Theta_{\mathrm{L}}$

Proof. Construct a Lyapunov functional $\mathrm{U}_{1}^{\mathrm{L}}\left(\mathrm{T}, \mathrm{C}^{*}, \mathrm{~T}^{*}, \mathrm{~V}\right)$ as follows:

$$
\begin{aligned}
\mathrm{U}_{1}^{\mathrm{L}}\left(\mathrm{T}, \mathrm{C}^{*}, \mathrm{~T}^{*}, V\right)= & \mathrm{T}-\mathrm{T}_{1}-\int_{\mathrm{T}_{1}}^{\mathrm{T}} \frac{\mathrm{g}\left(\mathrm{T}_{1}\right)}{\mathrm{g}(\vartheta)} \mathrm{d} \vartheta+\frac{\sigma}{\pi \rho+\sigma}\left(\mathrm{C}^{*}-\mathrm{C}_{1}^{*}-\int_{\mathrm{C}_{1}^{*}}^{\mathrm{C}^{*}} \frac{\psi\left(\mathrm{C}_{1}^{*}\right)}{\psi(\vartheta)} \mathrm{d} \vartheta\right) \\
& +\frac{\rho+\sigma}{\pi \rho+\sigma}\left(\mathrm{T}^{*}-\mathrm{T}_{1}^{*}-\int_{\mathrm{T}_{1}^{*}}^{\mathrm{T}^{*}} \frac{\xi\left(\mathrm{T}_{1}^{*}\right)}{\xi(\vartheta)} \mathrm{d} \vartheta\right)+\frac{g\left(\mathrm{~T}_{1}\right) \mathrm{h}_{1}\left(\mathrm{~V}_{1}\right)}{\mathrm{c} \varphi\left(\mathrm{V}_{1}\right)}\left(\mathrm{V}-\mathrm{V}_{1}-\int_{\mathrm{V}_{1}}^{V} \frac{\varphi(\mathrm{V})}{\varphi(\vartheta)} \mathrm{d} \vartheta\right) .
\end{aligned}
$$

It is seen that, $\mathrm{U}_{1}^{\mathrm{L}}\left(\mathrm{T}, \mathrm{C}^{*}, \mathrm{~T}^{*}, V\right)>0$ for all $\mathrm{T}, \mathrm{C}^{*}, \mathrm{~T}^{*}, V>0$, while $\mathrm{U}_{1}^{\mathrm{L}}\left(\mathrm{T}, \mathrm{C}^{*}, \mathrm{~T}^{*}, \mathrm{~V}\right)$ reaches its global minimum at $P_{1}$. Calculate $\frac{d U_{1}^{L}}{d t}$ as:

$$
\begin{aligned}
\frac{d u_{1}^{L}}{d t}= & \left(1-\frac{g\left(T_{1}\right)}{g(T)}\right)\left(f(T)-g(T) h_{1}(V)-g(T) h_{2}\left(T^{*}\right)\right) \\
& +\frac{\sigma}{\pi \rho+\sigma}\left(1-\frac{\psi\left(C_{1}^{*}\right)}{\psi\left(C^{*}\right)}\right)\left((1-\pi)\left(g(T) h_{1}(V)+g(T) h_{2}\left(T^{*}\right)\right)-(\rho+\sigma) \psi\left(C^{*}\right)\right) \\
& +\frac{\rho+\sigma}{\pi \rho+\sigma}\left(1-\frac{\xi\left(T_{1}^{*}\right)}{\xi\left(T^{*}\right)}\right)\left(\pi\left(g(T) h_{1}(V)+g(T) h_{2}\left(T^{*}\right)\right)+\sigma \psi\left(C^{*}\right)-\mu \xi\left(T^{*}\right)\right) \\
& +\frac{g\left(T_{1}\right) h_{1}\left(V_{1}\right)}{c \varphi\left(V_{1}\right)}\left(1-\frac{\varphi\left(V_{1}\right)}{\varphi(V)}\right)\left(b \xi\left(T^{*}\right)-c \varphi(V)\right) \\
= & \left(1-\frac{g\left(T_{1}\right)}{g(T)}\right)\left(f(T)-f\left(T_{1}\right)\right)+\left(1-\frac{g\left(T_{1}\right)}{g(T)}\right) f\left(T_{1}\right)+g\left(T_{1}\right) h_{1}(V)+g\left(T_{1}\right) h_{2}\left(T^{*}\right) \\
& -\frac{\sigma(1-\pi)}{\pi \rho+\sigma} \frac{\psi\left(C_{1}^{*}\right)}{\psi\left(C^{*}\right)}\left(g(T) h_{1}(V)+g(T) h_{2}\left(T^{*}\right)\right)+\frac{\sigma(\rho+\sigma)}{\pi \rho+\sigma} \psi\left(C_{1}^{*}\right)-\mu \frac{\rho+\sigma}{\pi \rho+\sigma} \xi\left(T^{*}\right) \\
& -\frac{\pi(\rho+\sigma)}{\pi \rho+\sigma} \frac{\xi\left(T_{1}^{*}\right)}{\xi\left(T^{*}\right)}\left(g(T) h_{1}(V)+g(T) h_{2}\left(T^{*}\right)\right)-\frac{\sigma(\rho+\sigma)}{\pi \rho+\sigma} \frac{\xi\left(T_{1}^{*}\right)}{\xi\left(T^{*}\right)} \psi\left(C^{*}\right)+\mu \frac{\rho+\sigma}{\pi \rho+\sigma} \xi\left(T_{1}^{*}\right) \\
& +b \frac{g\left(T_{1}\right) h_{1}\left(V_{1}\right)}{c \varphi\left(V_{1}\right)} \xi\left(T^{*}\right)-\frac{g\left(T_{1}\right) h_{1}\left(V_{1}\right)}{\varphi\left(V_{1}\right)} \varphi(V)-b \frac{g\left(T_{1}\right) h_{1}\left(V_{1}\right)}{c \varphi(V)} \xi\left(T^{*}\right)+g\left(T_{1}\right) h_{1}\left(V_{1}\right) .
\end{aligned}
$$

Collecting terms of (3.14) and applying the conditions of $P_{1}$ :

$$
\begin{aligned}
f\left(T_{1}\right) & =g\left(T_{1}\right) h_{1}\left(V_{1}\right)+g\left(T_{1}\right) h_{2}\left(T_{1}^{*}\right), \\
b \xi\left(T_{1}^{*}\right) & =c \varphi\left(V_{1}\right), \\
\mu \xi\left(T_{1}^{*}\right) & =\frac{(\pi \rho+\sigma)}{(\rho+\sigma)}\left(g\left(T_{1}\right) h_{1}\left(V_{1}\right)+g\left(T_{1}\right) h_{2}\left(T_{1}^{*}\right)\right), \\
c \varphi\left(V_{1}\right) & =\frac{b(\pi \rho+\sigma)}{\mu(\rho+\sigma)}\left(g\left(T_{1}\right) h_{1}\left(V_{1}\right)+g\left(T_{1}\right) h_{2}\left(T_{1}^{*}\right)\right), \\
\psi\left(C_{1}^{*}\right) & =\frac{(1-\pi)}{(\rho+\sigma)}\left(g\left(T_{1}\right) h_{1}\left(V_{1}\right)+g\left(T_{1}\right) h_{2}\left(T_{1}^{*}\right)\right),
\end{aligned}
$$

we get

$$
\begin{aligned}
\frac{d U_{1}^{L}}{d t}= & \left(1-\frac{g\left(T_{1}\right)}{g(T)}\right)\left(f(T)-f\left(T_{1}\right)\right)+\left(1-\frac{g\left(T_{1}\right)}{g(T)}\right)\left(g\left(T_{1}\right) h_{1}\left(V_{1}\right)+g\left(T_{1}\right) h_{2}\left(T_{1}^{*}\right)\right) \\
& +g\left(T_{1}\right) h_{1}(V)+g\left(T_{1}\right) h_{2}\left(T^{*}\right)-\frac{\sigma(1-\pi)}{\pi \rho+\sigma} g\left(T_{1}\right) h_{1}\left(V_{1}\right) \frac{\psi\left(C_{1}^{*}\right) g(T) h_{1}(V)}{\psi\left(C^{*}\right) g\left(T_{1}\right) h_{1}\left(V_{1}\right)}
\end{aligned}
$$




$$
\begin{aligned}
& -\frac{\sigma(1-\pi)}{\pi \rho+\sigma} g\left(T_{1}\right) h_{2}\left(T_{1}^{*}\right) \frac{\psi\left(C_{1}^{*}\right) g(T) h_{2}\left(T^{*}\right)}{\psi\left(C^{*}\right) g\left(T_{1}\right) h_{2}\left(T_{1}^{*}\right)}+\frac{\sigma(1-\pi)}{\pi \rho+\sigma}\left(g\left(T_{1}\right) h_{1}\left(V_{1}\right)+g\left(T_{1}\right) h_{2}\left(T_{1}^{*}\right)\right) \\
& +\left[b \frac{g\left(T_{1}\right) h_{1}\left(V_{1}\right)}{c \varphi\left(V_{1}\right)}-\mu \frac{\rho+\sigma}{\pi \rho+\sigma}\right] \xi\left(T^{*}\right)-\frac{\pi(\rho+\sigma)}{\pi \rho+\sigma} g\left(T_{1}\right) h_{1}\left(V_{1}\right) \frac{\xi\left(T_{1}^{*}\right) g(T) h_{1}(V)}{\xi\left(T^{*}\right) g\left(T_{1}\right) h_{1}\left(V_{1}\right)} \\
& -\frac{\pi(\rho+\sigma)}{\pi \rho+\sigma} g\left(T_{1}\right) h_{2}\left(T_{1}^{*}\right) \frac{\xi\left(T_{1}^{*}\right) g(T) h_{2}\left(T^{*}\right)}{\xi\left(T^{*}\right) g\left(T_{1}\right) h_{2}\left(T_{1}^{*}\right)}-\frac{\sigma(\rho+\sigma)}{\pi \rho+\sigma} \psi\left(C_{1}^{*}\right) \frac{\xi\left(T_{1}^{*}\right) \psi\left(C^{*}\right)}{\xi\left(T^{*}\right) \psi\left(C_{1}^{*}\right)}+g\left(T_{1}\right) h_{1}\left(V_{1}\right) \\
& +g\left(T_{1}\right) h_{2}\left(T_{1}^{*}\right)-g\left(T_{1}\right) h_{1}\left(V_{1}\right) \frac{\varphi(V)}{\varphi\left(V_{1}\right)}-g\left(T_{1}\right) h_{1}\left(V_{1}\right) \frac{\varphi\left(V_{1}\right) \xi\left(T^{*}\right)}{\varphi(V) \xi\left(T_{1}^{*}\right)}+g\left(T_{1}\right) h_{1}\left(V_{1}\right) \\
& =\left(1-\frac{g\left(T_{1}\right)}{g(T)}\right)\left(f(T)-f\left(T_{1}\right)\right)+\left(1-\frac{g\left(T_{1}\right)}{g(T)}\right)\left(g\left(T_{1}\right) h_{1}\left(V_{1}\right)+g\left(T_{1}\right) h_{2}\left(T_{1}^{*}\right)\right) \\
& +g\left(T_{1}\right) h_{1}\left(V_{1}\right)\left[\frac{h_{1}(V)}{h_{1}\left(V_{1}\right)}-\frac{\varphi(V)}{\varphi\left(V_{1}\right)}\right]+g\left(T_{1}\right) h_{2}\left(T_{1}^{*}\right)\left[\frac{h_{2}\left(T^{*}\right)}{h_{2}\left(T_{1}^{*}\right)}-\frac{\xi\left(T^{*}\right)}{\xi\left(T_{1}^{*}\right)}\right] \\
& -\frac{\sigma(1-\pi)}{\pi \rho+\sigma} g\left(T_{1}\right) h_{1}\left(V_{1}\right) \frac{\psi\left(C_{1}^{*}\right) g(T) h_{1}(V)}{\psi\left(C^{*}\right) g\left(T_{1}\right) h_{1}\left(V_{1}\right)}-\frac{\sigma(1-\pi)}{\pi \rho+\sigma} g\left(T_{1}\right) h_{2}\left(T_{1}^{*}\right) \frac{\psi\left(C_{1}^{*}\right) g(T) h_{2}\left(T^{*}\right)}{\psi\left(C^{*}\right) g\left(T_{1}\right) h_{2}\left(T_{1}^{*}\right)} \\
& +\frac{\sigma(1-\pi)}{\pi \rho+\sigma}\left(g\left(T_{1}\right) h_{1}\left(V_{1}\right)+g\left(T_{1}\right) h_{2}\left(T_{1}^{*}\right)\right)+\left[\frac{g\left(T_{1}\right) h_{2}\left(T_{1}^{*}\right)}{\xi\left(T_{1}^{*}\right)}+\frac{b g\left(T_{1}\right) h_{1}\left(V_{1}\right)}{c \varphi\left(V_{1}\right)}-\mu \frac{\rho+\sigma}{\pi \rho+\sigma}\right] \xi\left(T^{*}\right) \\
& -\frac{\pi(\rho+\sigma)}{\pi \rho+\sigma} g\left(T_{1}\right) h_{1}\left(V_{1}\right) \frac{\xi\left(T_{1}^{*}\right) g(T) h_{1}(V)}{\xi\left(T^{*}\right) g\left(T_{1}\right) h_{1}\left(V_{1}\right)}-\frac{\pi(\rho+\sigma)}{\pi \rho+\sigma} g\left(T_{1}\right) h_{2}\left(T_{1}^{*}\right) \frac{\xi\left(T_{1}^{*}\right) g(T) h_{2}\left(T^{*}\right)}{\xi\left(T^{*}\right) g\left(T_{1}\right) h_{2}\left(T_{1}^{*}\right)} \\
& -\sigma \frac{(1-\pi)}{\pi \rho+\sigma}\left(g\left(T_{1}\right) h_{1}\left(V_{1}\right)+g\left(T_{1}\right) h_{2}\left(T_{1}^{*}\right)\right) \frac{\xi\left(T_{1}^{*}\right) \psi\left(C^{*}\right)}{\xi\left(T^{*}\right) \psi\left(C_{1}^{*}\right)}+g\left(T_{1}\right) h_{1}\left(V_{1}\right)+g\left(T_{1}\right) h_{2}\left(T_{1}^{*}\right) \\
& -g\left(T_{1}\right) h_{1}\left(V_{1}\right) \frac{\varphi\left(V_{1}\right)}{\xi\left(T_{1}^{*}\right)} \frac{\xi\left(T^{*}\right)}{\varphi(V)}+g\left(T_{1}\right) h_{1}\left(V_{1}\right) .
\end{aligned}
$$

Equation (3.15) can be simplified as:

$$
\begin{aligned}
\frac{d u_{1}^{L}}{d t}= & \left(1-\frac{g\left(T_{1}\right)}{g(T)}\right)\left(f(T)-f\left(T_{1}\right)\right)+g\left(T_{1}\right) h_{1}\left(V_{1}\right)\left[\frac{h_{1}(V)}{h_{1}\left(V_{1}\right)}-1-\frac{\varphi(V)}{\varphi\left(V_{1}\right)}+\frac{h_{1}\left(V_{1}\right) \varphi(V)}{h_{1}(V) \varphi\left(V_{1}\right)}\right] \\
& +g\left(T_{1}\right) h_{2}\left(T_{1}^{*}\right)\left[\frac{h_{2}\left(T^{*}\right)}{h_{2}\left(T_{1}^{*}\right)}-1-\frac{\xi\left(T^{*}\right)}{\xi\left(T_{1}^{*}\right)}+\frac{h_{2}\left(T_{1}^{*}\right) \xi\left(T^{*}\right)}{h_{2}\left(T^{*}\right) \xi\left(T_{1}^{*}\right)}\right] \\
& +\frac{\sigma(1-\pi)}{\pi \rho+\sigma} g\left(T_{1}\right) h_{1}\left(V_{1}\right)\left[5-\frac{g\left(T_{1}\right)}{g(T)}-\frac{h_{1}\left(V_{1}\right) \varphi(V)}{h_{1}(V) \varphi\left(V_{1}\right)}-\frac{\psi\left(C_{1}^{*}\right) g(T) h_{1}(V)}{\psi\left(C^{*}\right) g\left(T_{1}\right) h_{1}\left(V_{1}\right)}-\frac{\varphi\left(V_{1}\right)}{\xi\left(T_{1}^{*}\right)} \frac{\xi\left(T^{*}\right)}{\varphi(V)}\right. \\
& \left.-\frac{\xi\left(T_{1}^{*}\right)}{\psi\left(C_{1}^{*}\right)} \frac{\psi\left(C^{*}\right)}{\xi\left(T^{*}\right)}\right] \\
& +\frac{\pi(\rho+\sigma)}{\pi \rho+\sigma} g\left(T_{1}\right) h_{1}\left(V_{1}\right)\left[4-\frac{g\left(T_{1}\right)}{g(T)}-\frac{h_{1}\left(V_{1}\right) \varphi(V)}{h_{1}(V) \varphi\left(V_{1}\right)}-\frac{\xi\left(T_{1}^{*}\right) g(T) h_{1}(V)}{\xi\left(T^{*}\right) g\left(T_{1}\right) h_{1}\left(V_{1}\right)}-\frac{\varphi\left(V_{1}\right)}{\xi\left(T_{1}^{*}\right)} \frac{\xi\left(T^{*}\right)}{\varphi(V)}\right] \\
& +\frac{\sigma(1-\pi)}{\pi \rho+\sigma} g\left(T_{1}\right) h_{2}\left(T_{1}^{*}\right)\left[4-\frac{g\left(T_{1}\right)}{g(T)}-\frac{h_{2}\left(T_{1}^{*}\right) \xi\left(T^{*}\right)}{h_{2}\left(T^{*}\right) \xi\left(T_{1}^{*}\right)}-\frac{\psi\left(C_{1}^{*}\right) g(T) h_{2}\left(T^{*}\right)}{\psi\left(C^{*}\right) g\left(T_{1}\right) h_{2}\left(T_{1}^{*}\right)}-\frac{\xi\left(T_{1}^{*}\right) \psi\left(C^{*}\right)}{\xi\left(T^{*}\right) \psi\left(C_{1}^{*}\right)}\right] \\
& +\frac{\pi(\rho+\sigma)}{\pi \rho+\sigma} g\left(T_{1}\right) h_{2}\left(T_{1}^{*}\right)\left[3-\frac{g\left(T_{1}\right)}{g(T)}-\frac{h_{2}\left(T_{1}^{*}\right) \xi\left(T^{*}\right)}{h_{2}\left(T^{*}\right) \xi\left(T_{1}^{*}\right)}-\frac{\xi\left(T_{1}^{*}\right) g(T) h_{2}\left(T^{*}\right)}{\xi\left(T^{*}\right) g\left(T_{1}\right) h_{2}\left(T_{1}^{*}\right)}\right] .
\end{aligned}
$$

Then, we obtain:

$$
\begin{aligned}
\frac{d U_{1}^{L}}{d t}= & \left(1-\frac{g\left(T_{1}\right)}{g(T)}\right)\left(f(T)-f\left(T_{1}\right)\right)+g\left(T_{1}\right) h_{1}\left(V_{1}\right)\left(\frac{h_{1}(V)}{h_{1}\left(V_{1}\right)}-\frac{\varphi(V)}{\varphi\left(V_{1}\right)}\right)\left(1-\frac{h_{1}\left(V_{1}\right)}{h_{1}(V)}\right) \\
& +g\left(T_{1}\right) h_{2}\left(T_{1}^{*}\right)\left(\frac{h_{2}\left(T^{*}\right)}{h_{2}\left(T_{1}^{*}\right)}-\frac{\xi\left(T^{*}\right)}{\xi\left(T_{1}^{*}\right)}\right)\left(1-\frac{h_{2}\left(T_{1}^{*}\right)}{h_{2}\left(T^{*}\right)}\right)
\end{aligned}
$$




$$
\begin{aligned}
& +\frac{\sigma(1-\pi)}{\pi \rho+\sigma} g\left(T_{1}\right) h_{1}\left(V_{1}\right)\left[5-\frac{g\left(T_{1}\right)}{g(T)}-\frac{h_{1}\left(V_{1}\right) \varphi(V)}{h_{1}(V) \varphi\left(V_{1}\right)}-\frac{\psi\left(C_{1}^{*}\right) g(T) h_{1}(V)}{\psi\left(C^{*}\right) g\left(T_{1}\right) h_{1}\left(V_{1}\right)}-\frac{\varphi\left(V_{1}\right)}{\xi\left(T_{1}^{*}\right)} \frac{\xi\left(T^{*}\right)}{\varphi(V)}\right. \\
& \left.-\frac{\xi\left(T_{1}^{*}\right)}{\psi\left(C_{1}^{*}\right)} \frac{\psi\left(C^{*}\right)}{\xi\left(T^{*}\right)}\right] \\
& +\frac{\pi(\rho+\sigma)}{\pi \rho+\sigma} g\left(T_{1}\right) h_{1}\left(V_{1}\right)\left[4-\frac{g\left(T_{1}\right)}{g(T)}-\frac{h_{1}\left(V_{1}\right) \varphi(V)}{h_{1}(V) \varphi\left(V_{1}\right)}-\frac{\xi\left(T_{1}^{*}\right) g(T) h_{1}(V)}{\xi\left(T^{*}\right) g\left(T_{1}\right) h_{1}\left(V_{1}\right)}-\frac{\varphi\left(V_{1}\right)}{\xi\left(T_{1}^{*}\right)} \frac{\xi\left(T^{*}\right)}{\varphi(V)}\right] \\
& +\frac{\sigma(1-\pi)}{\pi \rho+\sigma} g\left(T_{1}\right) h_{2}\left(T_{1}^{*}\right)\left[4-\frac{g\left(T_{1}\right)}{g(T)}-\frac{h_{2}\left(T_{1}^{*}\right) \xi\left(T^{*}\right)}{h_{2}\left(T^{*}\right) \xi\left(T_{1}^{*}\right)}-\frac{\psi\left(C_{1}^{*}\right) g(T) h_{2}\left(T^{*}\right)}{\psi\left(C^{*}\right) g\left(T_{1}\right) h_{2}\left(T_{1}^{*}\right)}-\frac{\xi\left(T_{1}^{*}\right) \psi\left(C^{*}\right)}{\xi\left(T^{*}\right) \psi\left(C_{1}^{*}\right)}\right] \\
& +\frac{\pi(\rho+\sigma)}{\pi \rho+\sigma} g\left(T_{1}\right) h_{2}\left(T_{1}^{*}\right)\left[3-\frac{g\left(T_{1}\right)}{g(T)}-\frac{h_{2}\left(T_{1}^{*}\right) \xi\left(T^{*}\right)}{h_{2}\left(T^{*}\right) \xi\left(T_{1}^{*}\right)}-\frac{\xi\left(T_{1}^{*}\right) g(T) h_{2}\left(T^{*}\right)}{\xi\left(T^{*}\right) g\left(T_{1}\right) h_{2}\left(T_{1}^{*}\right)}\right] .
\end{aligned}
$$

From Assumptions A1, A2 and A3, and the relation between the geometrical and the arithmetical means we obtain that if $\mathcal{R}_{0}^{\mathrm{L}}>1$, then $\frac{\mathrm{du}_{1}^{\mathrm{L}}}{\mathrm{dt}} \leqslant 0$, for all $\mathrm{T}, \mathrm{C}^{*}, \mathrm{~T}^{*}, \mathrm{~V}>0$, where the equality occurs at the equilibrium $P_{1}$. LaSalle's invariance principle implies the global stability of $P_{1}$.

\section{Numerical simulations}

In this section, we consider two examples where Assumptions A1-A4 can be satisfied.

\subsection{Example 1}

We consider the model

$$
\begin{aligned}
& \dot{\mathrm{T}}=\rho-d T+\sigma T\left(1-\frac{T}{T_{\max }}\right)-\frac{\beta_{1} T^{n} V}{\left(\eta_{1}+T^{n}\right)\left(\eta_{2}+V\right)}-\frac{\beta_{2} T^{n} T^{*}}{\left(\eta_{1}+T^{n}\right)\left(\bar{\eta}_{2}+T^{*}\right)}, \\
& \dot{T}^{*}=\frac{\beta_{1} T^{n} V}{\left(\eta_{1}+T^{n}\right)\left(\eta_{2}+V\right)}+\frac{\beta_{2} T^{n} T^{*}}{\left(\eta_{1}+T^{n}\right)\left(\bar{\eta}_{2}+T^{*}\right)}-\mu\left(c_{1} T^{*}+\bar{c}_{1} T^{* 2}\right), \\
& \dot{V}=b\left(c_{1} T^{*}+\bar{c}_{1} T^{* 2}\right)-c\left(c_{2} V+\bar{c}_{2} V^{2}\right) .
\end{aligned}
$$

We assume that $\sigma<d$ (see e.g. [5]) and $\rho, d, \sigma, \beta_{1}, \beta_{2}, n, \eta_{1}, \eta_{2}, \bar{\eta}_{2}, \mu, c_{1}, \bar{c}_{1}, c_{2}, \bar{c}_{2}>0$. In this example we consider the following:

$$
\begin{aligned}
f(T) & =\rho-d T+\sigma T\left(1-\frac{T}{T_{\max }}\right), & g(T) & =\frac{T^{n}}{\eta_{1}+T^{n}}, \\
h_{1}(V) & =\frac{\beta_{1} V}{\eta_{2}+V^{\prime}}, & h_{2}\left(T^{*}\right) & =\frac{\beta_{2} T^{*}}{\bar{\eta}_{2}+T^{*}}, \\
\xi\left(T^{*}\right) & =c_{1} T^{*}+\bar{c}_{1} T^{* 2}, & \varphi(V) & =c_{2} V+\bar{c}_{2} V^{2} .
\end{aligned}
$$

Now, we verify Assumptions A1-A3. We have $f\left(T_{0}\right)=0$, where $T_{0}=\frac{(\sigma-d) T_{\max }+\sqrt{(\sigma-d)^{2} T_{\max }^{2}+4 \sigma \rho T_{\max }}}{2 \sigma}>0$ and $f(0)=\rho>0$. Since $\sigma<d$, then

$$
f^{\prime}(T)=-d+\sigma-\frac{2 \sigma T}{T_{\max }}<0 .
$$

It follows that, $f(T)>0$ for all $T \in\left[0, T_{0}\right)$. Moreover, $f(T) \leqslant \rho-(d-\sigma) T=\rho-\bar{s} T$ for all $T \geqslant 0$ where $\bar{s}=\mathrm{d}-\sigma>0$. Thus, Assumption A1 is satisfied.

We have $g(T), h_{1}(V), h_{2}\left(T^{*}\right), \xi\left(T^{*}\right), \varphi(V)>0$ for all $T, T^{*}, V>0$ and $g(0)=h_{1}(0)=h_{2}(0)=\xi(0)=$ $\varphi(0)=0$. We have also

$$
g^{\prime}(T)=\frac{n \eta_{1} T^{n-1}}{\left(\eta_{1}+T^{n}\right)^{2}}>0, \quad \forall T>0,
$$




$$
\begin{aligned}
\mathrm{h}_{1}^{\prime}(\mathrm{V}) & =\frac{\beta_{1} \eta_{2}}{\left(\eta_{2}+\mathrm{V}\right)^{2}}>0, \quad \forall \mathrm{V} \geqslant 0, \\
\mathrm{~h}_{2}^{\prime}\left(\mathrm{T}^{*}\right) & =\frac{\beta_{2} \bar{\eta}_{2}}{\left(\bar{\eta}_{2}+\mathrm{T}^{*}\right)^{2}}>0, \quad \forall \mathrm{T}^{*} \geqslant 0, \\
\xi^{\prime}\left(\mathrm{T}^{*}\right) & =\mathrm{c}_{1}+2 \overline{\mathrm{c}}_{1} \mathrm{~T}^{*}>0, \quad \forall \mathrm{T}^{*} \geqslant 0, \\
\varphi^{\prime}(\mathrm{V}) & =\mathrm{c}_{2}+2 \overline{\mathrm{c}}_{2} \mathrm{~V}>0, \quad \forall \mathrm{V} \geqslant 0 .
\end{aligned}
$$

Moreover,

$$
\begin{aligned}
& \xi\left(\mathrm{T}^{*}\right)=\mathrm{c}_{1} \mathrm{~T}^{*}+\overline{\mathrm{c}}_{1} \mathrm{~T}^{* 2} \geqslant \mathrm{c}_{1} \mathrm{~T}^{*}, \quad \forall \mathrm{T}^{*} \geqslant 0, \\
& \varphi(\mathrm{V})=\mathrm{c}_{2} \mathrm{~V}+\overline{\mathrm{c}}_{2} \mathrm{~V}^{2} \geqslant \mathrm{c}_{2} \mathrm{~V}, \quad \forall \mathrm{V} \geqslant 0 .
\end{aligned}
$$

Then, Assumption A2 is satisfied. Now we verify Assumption A3. We have

$$
\begin{gathered}
\left(\frac{h_{1}(V)}{\varphi(V)}\right)^{\prime}=-\frac{\beta_{1}\left(c_{2}+2 \bar{c}_{2} V+\bar{c}_{2} \eta_{2}\right)}{\left(\eta_{2}+V\right)^{2}\left(c_{2}+\bar{c}_{2} V\right)^{2}}<0, \quad \forall V \geqslant 0, \\
\left(\frac{h_{2}\left(T^{*}\right)}{\xi\left(T^{*}\right)}\right)^{\prime}=-\frac{\beta_{2}\left(c_{1}+2 \bar{c}_{1} T^{*}+\bar{c}_{1} \bar{\eta}_{2}\right)}{\left(\bar{\eta}_{2}+T^{*}\right)^{2}\left(c_{1}+\bar{c}_{1} T^{*}\right)^{2}}<0, \quad \forall T^{*} \geqslant 0 .
\end{gathered}
$$

Thus, Assumption A3 is also satisfied.

The parameters $\mathcal{R}_{0}$ will be as follows:

$$
\mathcal{R}_{0}=\frac{\mathrm{T}_{0}^{\mathrm{n}}}{\mu\left(\eta_{1}+\mathrm{T}_{0}^{\mathrm{n}}\right)}\left(\frac{\mathrm{b} \beta_{1}}{\mathrm{cc}_{2} \eta_{2}}+\frac{\beta_{2}}{\mathrm{c}_{1} \bar{\eta}_{2}}\right),
$$

and then global stability results which are given by Theorems 2.6, 2.8 are compatible with our choices of the functions.

In the following we discuss the effect of the parameter $n$ on the parameter $\mathcal{R}_{0}$. We consider the following conditions

(C1) $\frac{b \beta_{1} c_{1} \bar{\eta}_{2}+\beta_{2} c c_{2} \eta_{2}}{\mu c c_{1} c_{2} \eta_{2} \bar{\eta}_{2}} \leqslant 1$;

(C2) $T_{0}=1$ and $\frac{b \beta_{1} c_{1} \bar{\eta}_{2}+\beta_{2} c c_{2} \eta_{2}}{\mu c c_{1} c_{2} \eta_{2} \bar{\eta}_{2}\left(\eta_{1}+1\right)} \leqslant \eta_{1}+1$;

(C3) $\mathrm{T}_{0}>1$ and $1<\frac{\mathrm{b} \beta_{1} c_{1} \bar{\eta}_{2}+\beta_{2} c c_{2} \eta_{2}}{\mu c c_{1} c_{2} \eta_{2} \bar{\eta}_{2}\left(\eta_{1}+1\right)}<\eta_{1}+1$;

(C4) $T_{0}<1$ and $\frac{b \beta_{1} c_{1} \bar{\eta}_{2}+\beta_{2} c c_{2} \eta_{2}}{\mu c c_{1} c_{2} \eta_{2} \bar{\eta}_{2}}>\eta_{1}+1$.

One can easily prove the following corollary:

Corollary 4.1. Let $\mathcal{R}_{0}$ be given by (4.4).

(i) if (C1) or (C2) is satisfied, then $\mathcal{R}_{0} \leqslant 1$ for all $\mathrm{n}>0$;

(ii) if (C3) or (C4) is satisfied, then there exists $\mathrm{N}>0$ such that $\mathcal{R}_{0} \leqslant 1$ for all $0<\mathrm{n} \leqslant \mathrm{N}$ and $\mathcal{R}_{0}>1$ for all $n>N$.

In order to illustrate our theoretical results, we perform numerical simulations for system (4.1), (4.2), (4.3) with parameters values given in Table 1 . In the figures we show the evolution of the three states $T, T^{*}$ and $\mathrm{V}$. We have used MATLAB for all computations. To show the global stability results we consider three different initial conditions as

IC1: $\mathrm{T}(0)=500, \mathrm{~T}^{*}(0)=30, \mathrm{~V}(0)=15$, 
IC2: $\mathrm{T}(0)=200, \mathrm{~T}^{*}(0)=3, \mathrm{~V}(0)=1.5$,

IC3: $\mathrm{T}(0)=30, \mathrm{~T}^{*}(0)=15, \mathrm{~V}(0)=9$.

Table 1: The data of system (4.1)-(4.3).

\begin{tabular}{cccccccc}
\hline Parameter & Value & Parameter & Value & Parameter & Value & Parameter & Value \\
\hline$\rho$ & 10 & $\beta_{1}$ & varied & $\bar{\eta}_{2}$ & 110 & $\mathrm{~b}$ & 1.2 \\
$\mathrm{~d}$ & 0.01 & $\mathrm{n}, \eta_{1}$ & varied & $\mu$ & 0.15 & $\mathrm{c}$ & 3.2 \\
$\sigma$ & 0.001 & $\eta_{2}$ & 140 & $\mathrm{c}_{1}$ & 0.9 & $\mathrm{c}_{2}$ & 1 \\
$\mathrm{~T}_{\max }$ & 1200 & $\beta_{2}$ & 0.001 & $\overline{\mathrm{c}}_{1}$ & 0.001 & $\overline{\mathrm{c}}_{2}$ & 0.002 \\
\hline
\end{tabular}

We investigate the stability of equilibria by varying three parameters $\beta_{1}, \eta_{1}$ and $n$, while the other parameters are fixed.

Case (1). $\eta_{1}=1.01, \mathrm{n}=1.003$ and $\beta_{1}$ is varied as:

(i) $\beta_{1}=20$, then we compute $\mathcal{R}_{0}=0.3569<1$. From Lemma 2.5 we have that the system has one equilibrium $P_{0}$. From Figures 1, 2, 3 we can see that, the concentration of uninfected CD4 ${ }^{+} \mathrm{T}$ cells is increasing and tends its normal value $\mathrm{T}_{0}=1015.6060$, while the concentrations of infected cells and free virus particles are decaying and approaching zero for all the three initial conditions IC1-IC3. It means that, $\mathrm{P}_{0}$ is globally asymptotically stable and the virus will be removed. This result supports the result of Theorem 2.6.

(ii) $\beta_{1}=70$, and then, $\mathcal{R}_{0}=1.2489>1$. Lemma 2.5 states that the system has two positive equilibria $P_{0}$ and $P_{1}$. It is clear from Figures 4, 5, 6 that, both the numerical results and the theoretical results given in Theorem 2.8 are consistent. It is seen that, the solutions of the system converge to the equilibrium $\mathrm{P}_{1}(38.5939,66.5683,23.0646)$ for all the three initial conditions IC1-IC3.

Case (2). $\beta_{1}=70, \eta_{1}=350$ and $n$ is varied. In this case, we consider the initial condition IC1. The values of $\mathcal{R}_{0}$ and the equilibria of system (4.1)-(4.3) with different values of $n$ are presented in Table 2.

Table 2: The values of equilibria and $\mathcal{R}_{0}$ for model (4.1)-(4.3) with different values of $n$.

\begin{tabular}{ccc}
\hline $\mathrm{n}$ & The equilibria & $\mathcal{R}_{0}$ \\
\hline 1 & $\mathrm{P}_{0}=(1015.6060,0,0)$ & 0.9297 \\
1.01 & $\mathrm{P}_{0}=(1015.6060,0,0)$ & 0.9459 \\
1.02 & $\mathrm{P}_{0}=(1015.6060,0,0)$ & 0.9615 \\
1.046324759 & $\mathrm{P}_{0}=(1015.6060,0,0)$ & 1 \\
1.5 & $\mathrm{P}_{1}=(287.9589,51.4264,17.7202)$ & 1.2367 \\
2 & $\mathrm{P}_{1}=(98.2145,63.0499,21.8180)$ & 1.2496 \\
3 & $\mathrm{P}_{1}=(24.5957,67.38531,23.3545)$ & 1.2501 \\
\hline
\end{tabular}

From Table 2 we can see that, the values of $\mathcal{R}_{0}$ is increased as $n$ is increased and the asymptotic properties of the equilibria are changed. Using the values of the parameters given in Table 1, we obtain that: $\mathrm{T}_{0}=1015.6060>1$ and $\frac{\mathrm{b} \beta_{1} c_{1} \bar{\eta}_{2}+\beta_{2} c c_{2} \eta_{2}}{\mu c c_{1} c_{2} \eta_{2} \bar{\eta}_{2}}=1.2501<\eta_{1}+1$, then Corollary 4.1 (ii) is satisfied and $\mathrm{N}=1.046324759$, thus as shown in Table 2 , we have

(i) if $0<\mathrm{n}<\mathrm{N}$, then $\mathrm{P}_{0}$ is GAS;

(ii) if $N \leqslant n$, then $P_{1}$ exists and it is GAS.

Figures 7, 8, 9 and Table 2 show that, when $n<N$, the trajectory of the system tends to the equilibrium $P_{0}$. Conversely, when $n>N$, then the trajectory will converge to $P_{1}$ as shown in the figures.

Case (3). $\beta_{1}=70, n=1$ and $\eta_{1}$ is varied.

In this case, we consider the initial condition IC1. We take the values $\beta_{1}=70$ and $n=1$. The values of $\mathcal{R}_{0}$ and the equilibrium of system (4.1)-(4.3) with different values of $\eta_{1}$ are presented in Table 3. 
Table 3: The values of equilibria and $\mathcal{R}_{0}$ for model (4.1)-(4.3) with different values of $\eta_{1}$.

\begin{tabular}{ccc}
\hline$\eta_{1}$ & The equilibria & $\mathcal{R}_{0}$ \\
\hline 1 & $\mathrm{P}_{1}=(38.5939,66.5682,23.0646)$ & 1.2488 \\
15 & $\mathrm{P}_{1}=(240.3507,54.4053,18.7673)$ & 1.2319 \\
70 & $\mathrm{P}_{1}=(550.4372,34.2078,11.7097)$ & 1.1695 \\
253.9698850 & $\mathrm{P}_{0}=(1015.6060,0,0)$ & 1 \\
270 & $\mathrm{P}_{0}=(1015.6060,0,0)$ & 0.9875 \\
300 & $\mathrm{P}_{0}=(1015.6060,0,0)$ & 0.9650 \\
\hline
\end{tabular}

From Table 3 we can see that, the values of $\mathcal{R}_{0}$ is decreased as $\eta_{1}$ is increased. Using the values of the parameters given in Table 1, we obtain that the following:

(i) if $1 \leqslant \eta_{1}<253.9698850$, then $P_{1}$ exists and it is GAS;

(ii) if $\eta_{1} \geqslant 253.9698850$, then $P_{0}$ is GAS. Figures 10, 11, 12 show that the numerical results are also compatible with the results of Theorems 2.6, 2.8.

\subsection{Example 2}

We consider the following model:

$$
\begin{aligned}
\dot{\mathrm{T}} & =\rho-d T+\sigma T\left(1-\frac{T}{T_{\max }}\right)-\frac{\beta_{1} T^{n} V}{\left(\eta_{1}+T^{n}\right)\left(\eta_{2}+V\right)}-\frac{\beta_{2} T^{n} T^{*}}{\left(\eta_{1}+T^{n}\right)\left(\bar{\eta}_{2}+T^{*}\right)}, \\
\dot{C}^{*} & =(1-\pi)\left(\frac{\beta_{1} T^{n} V}{\left(\eta_{1}+T^{n}\right)\left(\eta_{2}+V\right)}+\frac{\beta_{2} T^{n} T^{*}}{\left(\eta_{1}+T^{n}\right)\left(\bar{\eta}_{2}+T^{*}\right)}\right)-(\rho+\sigma)\left(c_{3} C^{*}+\bar{c}_{3} C^{* 2}\right), \\
\dot{T}^{*} & =\pi\left(\frac{\beta_{1} T^{n} V}{\left(\eta_{1}+T^{n}\right)\left(\eta_{2}+V\right)}+\frac{\beta_{2} T^{n} T^{*}}{\left(\eta_{1}+T^{n}\right)\left(\bar{\eta}_{2}+T^{*}\right)}\right)+\sigma\left(c_{3} C^{*}+\bar{c}_{3} C^{* 2}\right)-\mu\left(c_{1} T^{*}+\bar{c}_{1} T^{* 2}\right), \\
\dot{V} & =b\left(c_{1} T^{*}+\bar{c}_{1} T^{* 2}\right)-c\left(c_{2} V+\bar{c}_{2} V^{2}\right) .
\end{aligned}
$$

In this example the function $\psi$ is chosen as:

$$
\psi\left(C^{*}\right)=c_{3} C^{*}+\bar{c}_{3} C^{* 2},
$$

which satisfies Assumption A4. The basic reproduction number $\mathcal{R}_{0}^{\mathrm{L}}$ for model (4.5), (4.6), (4.7), (4.8) is given by

$$
\mathcal{R}_{0}^{\mathrm{L}}=\frac{(\pi \rho+\sigma) \mathrm{T}_{0}^{\mathrm{n}}}{\mu(\rho+\sigma)\left(\eta_{1}+T_{0}^{n}\right)}\left(\frac{\mathrm{b} \beta_{1}}{\mathrm{cc}_{2} \eta_{2}}+\frac{\beta_{2}}{c_{1} \bar{\eta}_{2}}\right) .
$$

We note that all the conditions of Theorems 3.4, 3.5 are satisfied.

In the following we discuss the effect of the parameter $n$ on $\mathcal{R}_{0}^{\mathrm{L}}$. Consider the following conditions:

(C5) $\frac{(\pi \rho+\sigma)\left(b \beta_{1} c_{1} \bar{\eta}_{2}+\beta_{2} c c_{2} \eta_{2}\right)}{\mu c c_{1} c_{2} \eta_{2} \bar{\eta}_{2}(\rho+\sigma)} \leqslant 1$;

(C6) $T_{0}=1$ and $\frac{(\pi \rho+\sigma)\left(b \beta_{1} c_{1} \bar{\eta}_{2}+\beta_{2} c c_{2} \eta_{2}\right)}{\mu c c_{1} c_{2} \eta_{2} \bar{\eta}_{2}(\rho+\sigma)} \leqslant \eta_{1}+1$;

(C7) $\mathrm{T}_{0}>1$ with $1<\frac{(\pi \rho+\sigma)\left(\mathrm{b} \beta_{1} c_{1} \bar{\eta}_{2}+\beta_{2} c_{c} \eta_{2}\right)}{\mu c c_{1} c_{2} \eta_{2} \bar{\eta}_{2}(\rho+\sigma)}<\eta_{1}+1$;

(C8) $\mathrm{T}_{0}<1$ with $\frac{(\pi \rho+\sigma)\left(\mathrm{b} \beta_{1} c_{1} \bar{\eta}_{2}+\beta_{2} c c_{2} \eta_{2}\right)}{\mu c c_{1} c_{2} \eta_{2} \bar{\eta}_{2}(\rho+\sigma)}>\eta_{1}+1$.

Corollary 4.2. Let $\mathcal{R}_{0}^{\mathrm{L}}$ be given by (4.9).

(i) if (C5) or (C6) is satisfied, then $\mathcal{R}_{0}^{\mathrm{L}} \leqslant 1$ for all $\mathrm{n}>0$;

(ii) if (C7) or (C8) is satisfied, then there exists $\mathrm{N}^{\mathrm{L}}>0$ such that $\mathcal{R}_{0}^{\mathrm{L}} \leqslant 1$ for all $0<\mathrm{n} \leqslant \mathrm{N}^{\mathrm{L}}$ and $\mathcal{R}_{0}^{\mathrm{L}}>1$ for all $n>N^{L}$. 
In order to illustrate our theoretical results, we perform numerical simulations for the model (4.5)-(4.8) with parameters values given in Table 4 . In the figures we show the evolution of the three states $T, C^{*}, T^{*}$ and $V$. We discuss the effect of the parameters, $\beta_{1}$ and $\pi$ on the dynamical behavior of the system in detail to investigate the theoretical results involved in Theorems 3.4, 3.5.

Table 4: The data of system (4.5)-(4.8).

\begin{tabular}{cccccccc}
\hline Parameter & Value & Parameter & Value & Parameter & Value & Parameter & Value \\
\hline$\rho$ & 10 & $\pi$ & varied & $\mathrm{c}_{1}$ & 0.9 & $\overline{\mathrm{c}}_{2}$ & 0.002 \\
$\mathrm{~d}$ & 0.01 & $\eta_{2}$ & 140 & $\overline{\mathrm{c}}_{1}$ & 0.001 & $\sigma$ & 0.05 \\
$\sigma$ & 0.001 & $\beta_{2}$ & 0.001 & $\mathrm{~b}$ & 1.2 & $\mathrm{c}_{3}$ & 1 \\
$\mathrm{~T}_{\max }$ & 1200 & $\bar{\eta}_{2}$ & 110 & $\mathrm{c}$ & 3.2 & $\overline{\mathrm{c}}_{3}$ & 0.001 \\
$\beta_{1}$ & varied & $\mu$ & 0.15 & $\rho$ & 0.1 & & \\
$\mathrm{n}$ & 1 & $\eta_{1}$ & 1 & $\mathrm{c}_{2}$ & 1 & & \\
\hline
\end{tabular}

To show the global stability of the equilibria we consider three different initial conditions:

IC4: $\mathrm{T}(0)=500, \mathrm{C}^{*}(0)=5, \mathrm{~T}^{*}(0)=40, \mathrm{~V}(0)=13$,

IC5: $\mathrm{T}(0)=200, \mathrm{C}^{*}(0)=30, \mathrm{~T}^{*}(0)=3, \mathrm{~V}(0)=1.5$,

IC6: $\mathrm{T}(0)=30, \mathrm{C}^{*}(0)=50, \mathrm{~T}^{*}(0)=15, \mathrm{~V}(0)=9$.

Case (1). $\pi=0.7$ and $\beta_{1}$ is varied as:

(i) $\beta_{1}=20$, then $\mathcal{R}_{0}^{\mathrm{L}}=0.2855<1$. From Lemma 3.3 we have that the system has one equilibrium $P_{0}$. From Figures 13, 14, 15, 16 we can see that, the concentration of uninfected cells is increasing and tends its normal value $\mathrm{T}_{0}=1015.6060$, while the concentrations of latently infected cells, productively infected cells and free virus particles are decaying and approaching zero for all the three initial conditions IC4-IC6. It means that, $\mathrm{P}_{0}$ is globally asymptotically stable and the virus will be removed. This result supports the result of Theorem 3.4.

(ii) $\beta_{1}=80$, then $\mathcal{R}_{0}^{\mathrm{L}}=1.1418>1$. Lemma 3.3 states that the system has two positive equilibria $P_{0}$ and $P_{1}$. It is clear from Figures 17, 18, 19, 20 that both the numerical results and the theoretical results given in Theorem 3.5 are consistent. It is seen that, the solutions of the system converge to the equilibrium $\mathrm{P}_{1}(258.1068,15.0175,43.1005,14.8047)$, for all the three initial conditions IC4-IC6.

Case (2). $\beta_{1}=80$ and $\pi$ is varied.

In this case, we consider the initial condition IC4. The values of $\mathcal{R}_{0}^{\mathrm{L}}$ and the equilibria of system (4.5)-(4.8) with different values of $\pi$ are presented in Table 5 .

Table 5: The values of steady states and $\mathcal{R}_{0}^{\mathrm{L}}$ for model (4.5)-(4.8) with different values of $\pi$.

\begin{tabular}{ccc}
\hline$\pi$ & The equilibria & $\mathcal{R}_{0}^{\mathrm{L}}$ \\
\hline 0.1 & $\mathrm{E}_{0}=(1015.6060,0,0,0)$ & 0.5709 \\
0.3 & $\mathrm{E}_{0}=(1015.6060,0,0,0)$ & 0.7612 \\
0.5509843243 & $\mathrm{E}_{0}=(1015.6060,0,0,0)$ & 1 \\
0.7 & $\mathrm{E}_{1}=(258.1068,15.0175,43.1005,14.8047)$ & 1.1418 \\
0.8 & $\mathrm{E}_{1}=(25.7432,12.8583,58.8575,20.3363)$ & 1.2369 \\
0.9 & $\mathrm{E}_{1}=(9.7632,6.5649,63.9796,22.1471)$ & 1.3321 \\
\hline
\end{tabular}

From Table 5 we can see that, the values of $\mathcal{R}_{0}^{\mathrm{L}}$ is increased as $\pi$ is increased. That is, the trajectory of system will converge to $P_{0}$ for small values of $\pi$ and they will converge to $P_{1}$ for larger values of $\pi$. Using the values of the parameters given in Table 4, we obtain the following: 
(i) if $0<\pi<0.5509843243$, then $\mathrm{P}_{0}$ is GAS;

(ii) if $\pi \geqslant 0.5509843243$, then $P_{1}$ exists and it is GAS.

We note that, when $\pi$ is very small, most of the infected cells will be latent and surely the converse is equally true. In this case, the virus particles will be extremely decayed and the population of the uninfected cells will be increased. This gives us some indications on suggesting new drugs to increase the latent part of the infected cells.

Figures 21, 22, 23, 24 show that the numerical results are also compatible with the results of Theorems 3.4, 3.5.

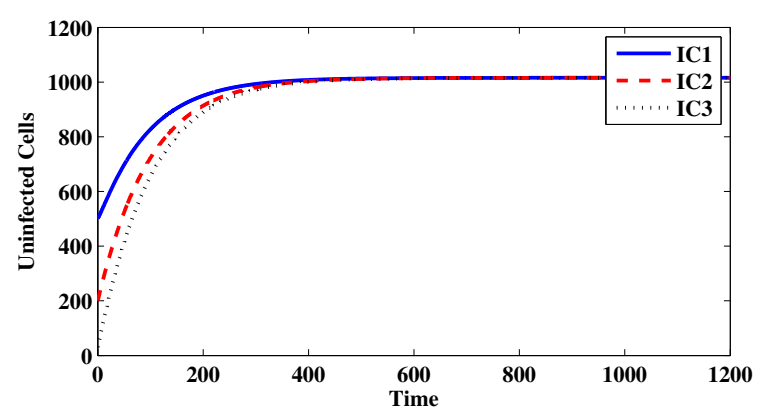

Figure 1: Evolution of uninfected cells for system (4.1)-(4.3) with initial IC1-IC3 in case of $\mathcal{R}_{0} \leqslant 1$.

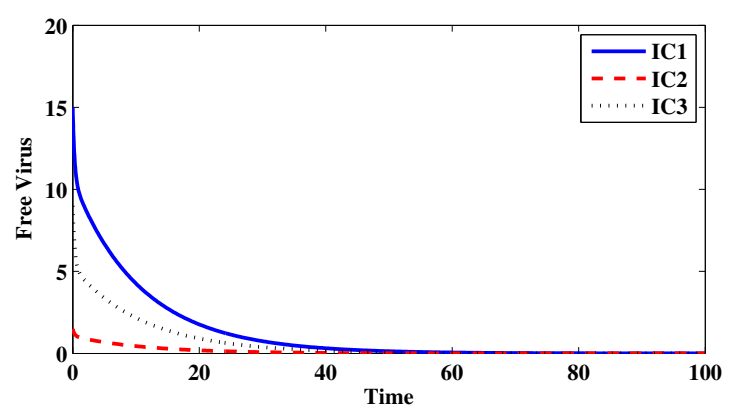

Figure 3: Evolution of free viruses for system (4.1)-(4.3) with initial IC1-IC3 in case of $\mathcal{R}_{0} \leqslant 1$.

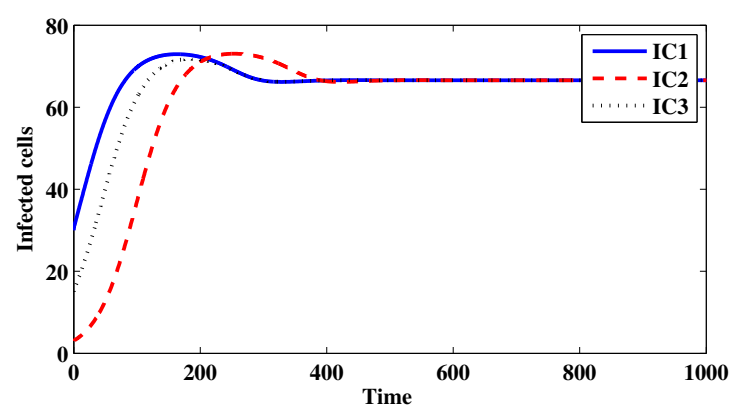

Figure 5: Evolution of infected cells for system (4.1)-(4.3) with initial IC1-IC3 in case of $\mathcal{R}_{0}>1$.

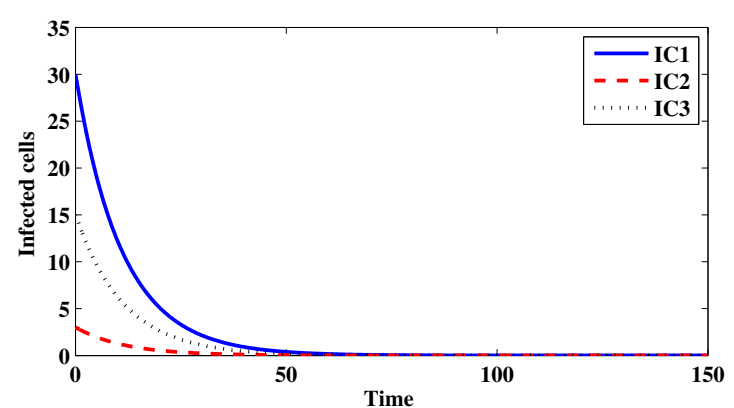

Figure 2: Evolution of infected cells for system (4.1)-(4.3) with initial IC1-IC3 in case of $\mathcal{R}_{0} \leqslant 1$.

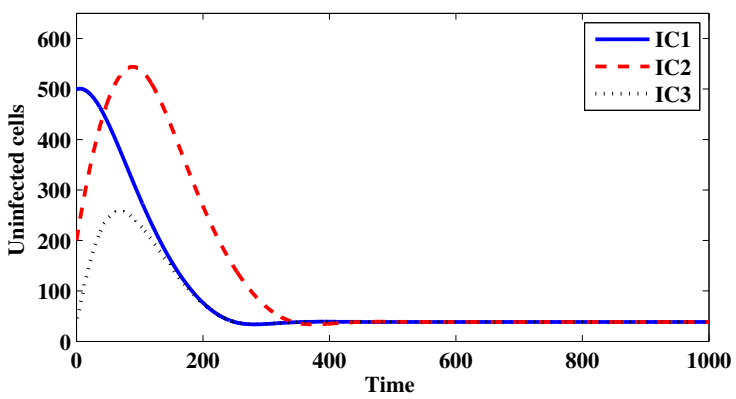

Figure 4: Evolution of uninfected cells for system (4.1)-(4.3) with initial IC1-IC3 in case of $\mathcal{R}_{0}>1$.

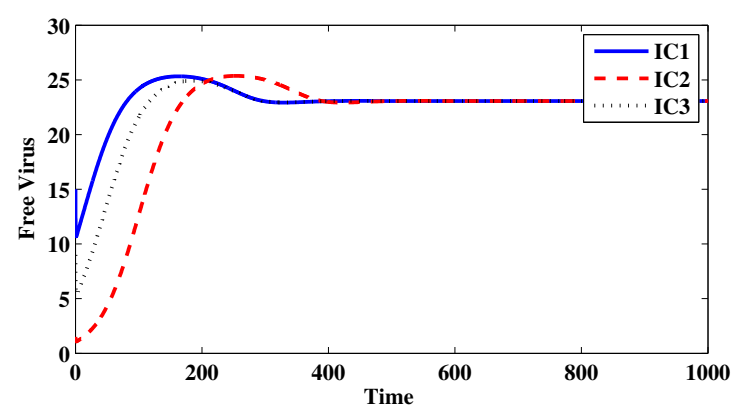

Figure 6: Evolution of free viruses for system (4.1)-(4.3) with initial IC1-IC3 in case of $\mathcal{R}_{0}>1$. 


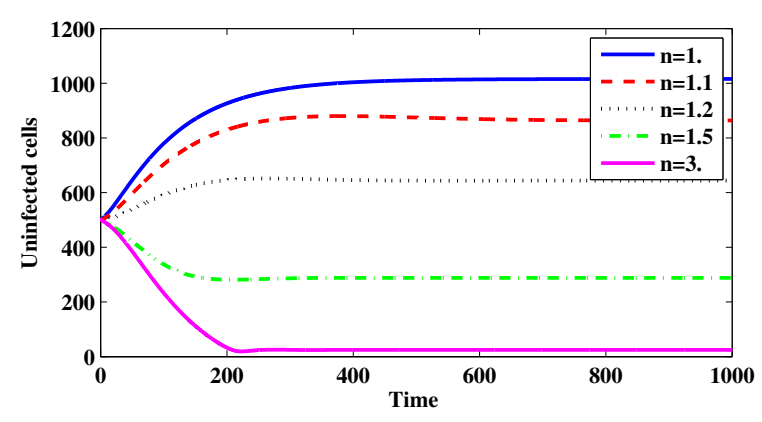

Figure 7: Evolution of uninfected cells for system (4.1)-(4.3) with different values of the parameter $n$.

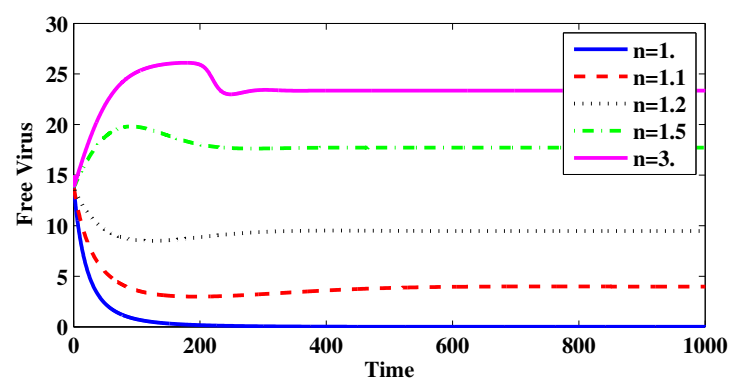

Figure 9: Evolution of free viruses for system (4.1)-(4.3) with different values of the parameter $n$.

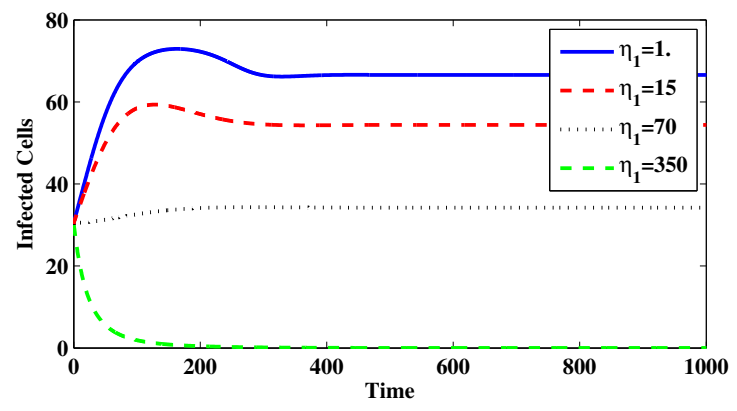

Figure 11: Evolution of infected cells for system (4.1)-(4.3) with different values of the parameter $\eta_{1}$.

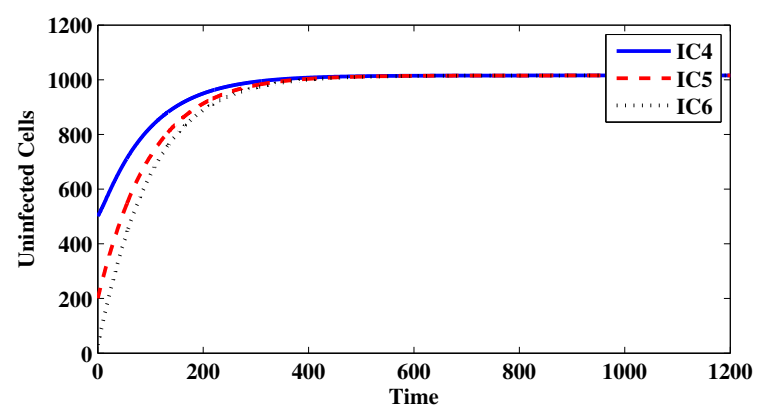

Figure 13: Evolution of uninfected cells for system (4.5)(4.8) with initial IC1-IC3 in case of $\mathcal{R}_{0}^{\mathrm{L}} \leqslant 1$.

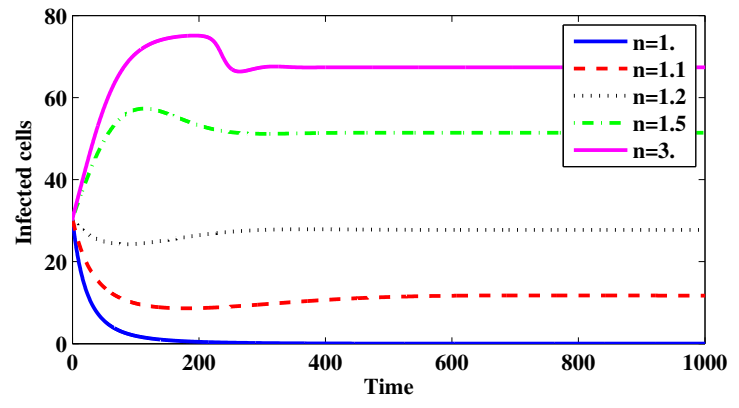

Figure 8: Evolution of infected cells for system (4.1)-(4.3) with different values of the parameter $n$.

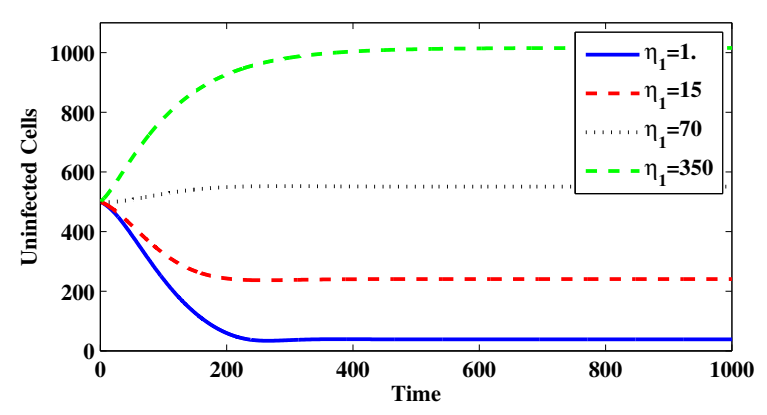

Figure 10: Evolution of uninfected cells for system (4.1)(4.3) with different values of the parameter $\eta_{1}$.

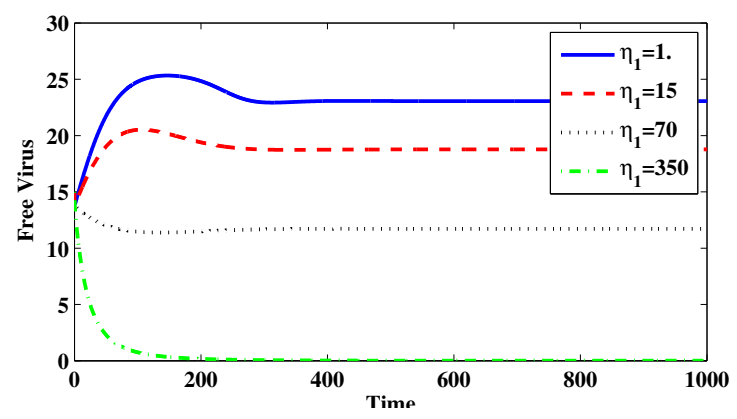

Figure 12: Evolution of free viruses for system (4.1)-(4.3) with different values of the parameter $\eta_{1}$.

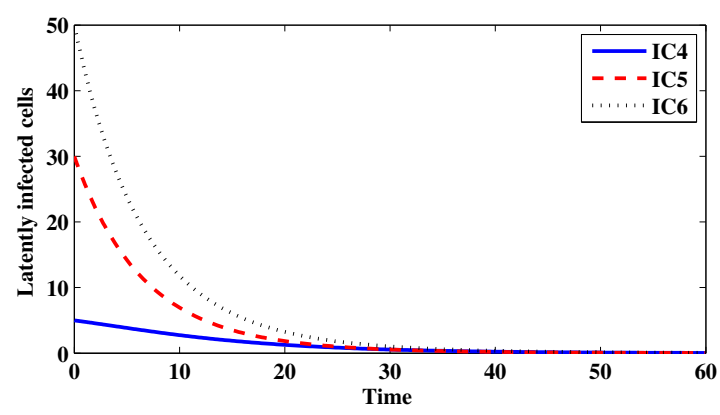

Figure 14: Evolution of latently infected cells for system (4.5)-(4.8) with initial IC1-IC3 in case of $\mathcal{R}_{0}^{\mathrm{L}} \leqslant 1$. 


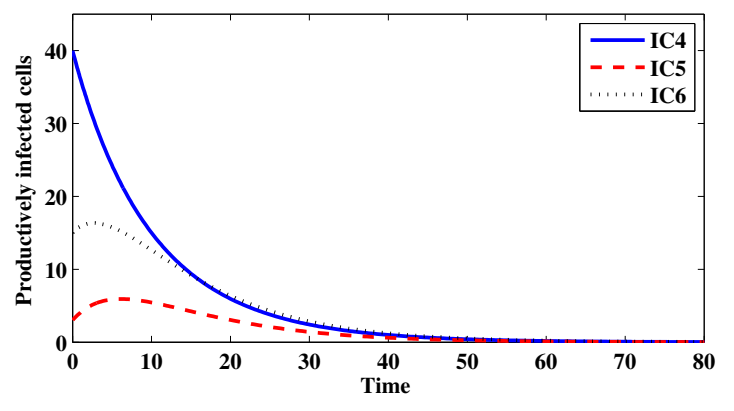

Figure 15: Evolution of productively infected cells for system (4.5)-(4.8) with initial IC1-IC3 in case of $\mathcal{R}_{0}^{\mathrm{L}} \leqslant 1$.

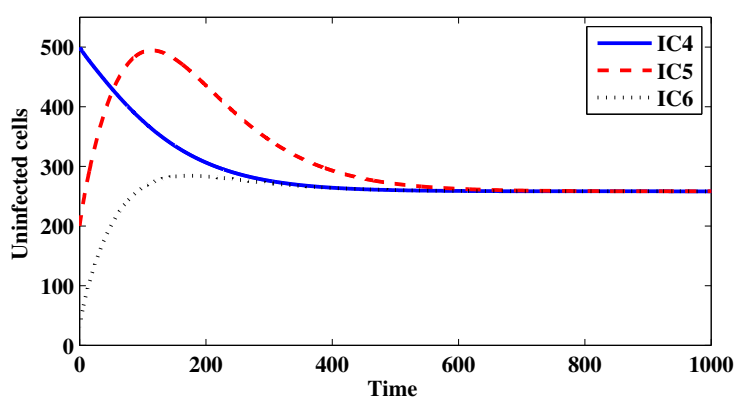

Figure 17: Evolution of uninfected cells for system (4.5)(4.8) with initial IC1-IC3 in case of $\mathcal{R}_{0}^{\mathrm{L}}>1$.

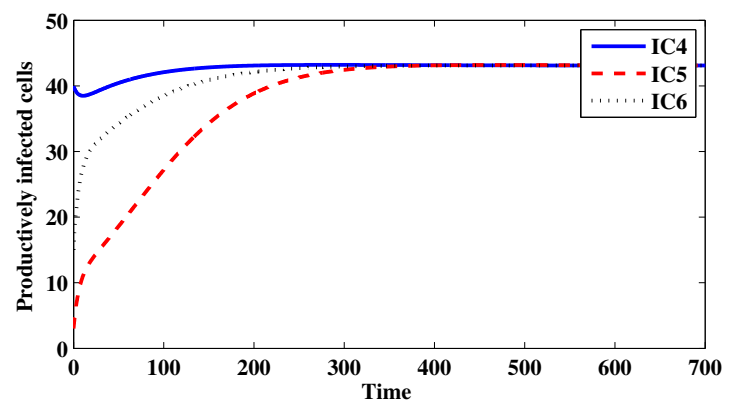

Figure 19: Evolution of productively infected cells for system (4.5)-(4.8) with initial IC1-IC3 in case of $\mathcal{R}_{0}^{\mathrm{L}}>1$.

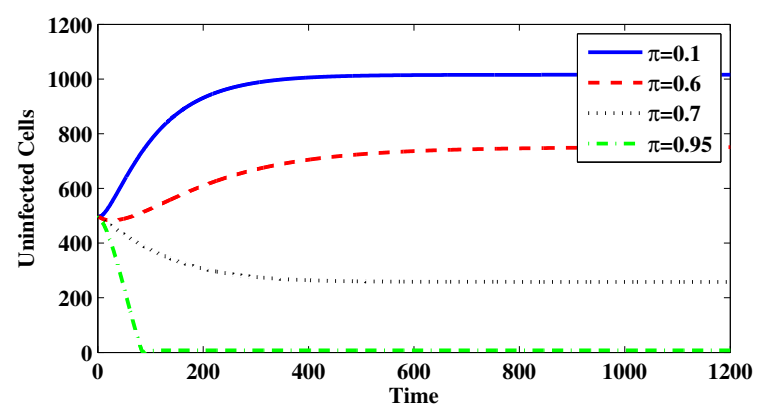

Figure 21: Evolution of uninfected cells for system (4.5)(4.8) with different values of the parameter $\pi$.

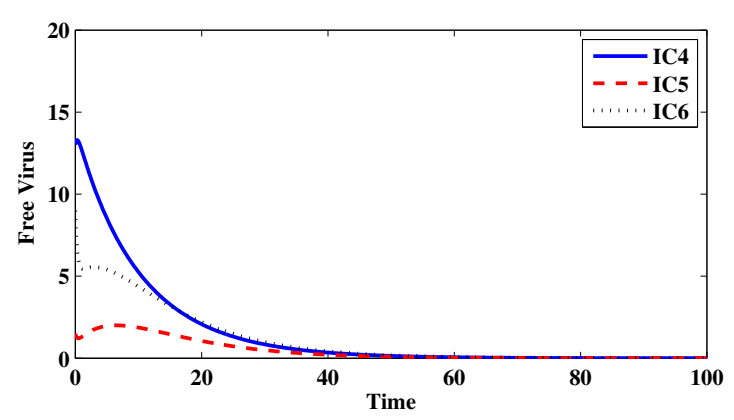

Figure 16: Evolution of free viruses for system (4.5)-(4.8) with initial IC1-IC3 in case of $\mathcal{R}_{0}^{\mathrm{L}} \leqslant 1$.

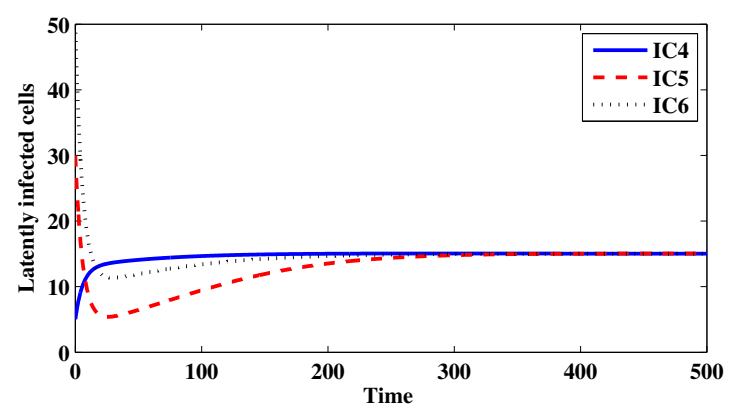

Figure 18: Evolution of latently infected cells for system (4.5)-(4.8) with initial IC1-IC3 in case of $\mathcal{R}_{0}^{\mathrm{L}}>1$.

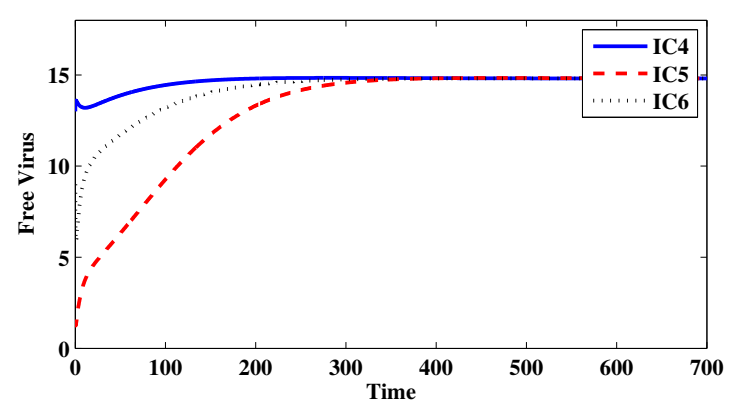

Figure 20: Evolution of free viruses for system (4.5)-(4.8) with initial IC1-IC3 in case of $\mathcal{R}_{0}^{\mathrm{L}}>1$.

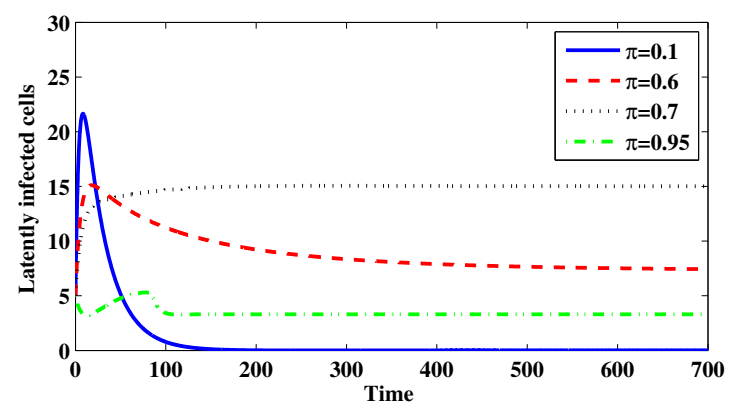

Figure 22: Evolution of latently infected cells for system (4.5)-(4.8) with different values of the parameter $\pi$. 


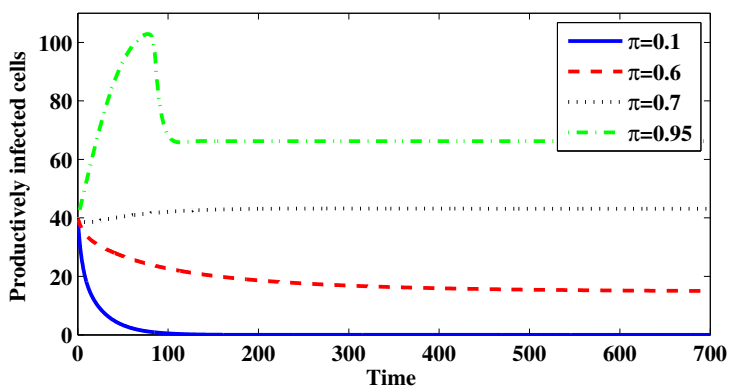

Figure 23: Evolution of productively infected cells for system (4.5)-(4.8) with different values of the parameter $\pi$.

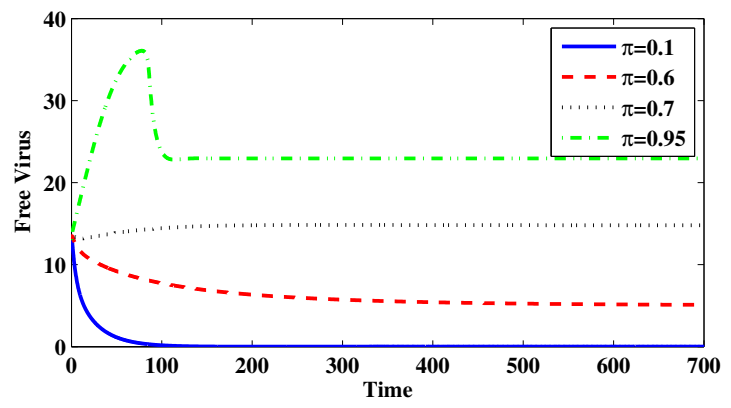

Figure 24: Evolution of free viruses for system (4.5)-(4.8) with different values of the parameter $\pi$.

\section{Acknowledgment}

This article was funded by the Deanship of Scientific Research (DSR), King Abdulaziz University, Jeddah. The authors, therefore, acknowledge with thanks DSR technical and financial support.

\section{References}

[1] N. Bairagi, D. Adak, Global analysis of HIV-1 dynamics with Hill type infection rate and intracellular delay, Appl. Math. Model., 38 (2014), 5047-5066. 1

[2] B. Buonomo, C. Vargas-De-León, Global stability for an HIV-1 infection model including an eclipse stage of infected cells, J. Math. Anal. Appl., 385 (2012), 709-720. 1

[3] S.-S. Chen, C.-Y. Cheng, Y. Takeuchi, Stability analysis in delayed within-host viral dynamics with both viral and cellular infections, J. Math. Anal. Appl., 442 (2016), 642-672. 1

[4] R. V. Culshaw, S.-G. Ruan, G. Webb, A mathematical model of cell-to-cell spread of HIV-1 that includes a time delay, J. Math. Biol., 46 (2003), 425-444. 1

[5] P. De Leenheer, H. L. Smith, Virus dynamics: a global analysis, SIAM J. Appl. Math., 63 (2003), 1313-1327. 4.1

[6] A. M. Elaiw, Global properties of a class of HIV models, Nonlinear Anal. Real World Appl., 11 (2010), 2253-2263. 1

[7] A. M. Elaiw, Global properties of a class of virus infection models with multitarget cells, Nonlinear Dynam., 69 (2012), 423-435.

[8] A. M. Elaiw, N. A. Almuallem, Global properties of delayed-HIV dynamics models with differential drug efficacy in cocirculating target cells, Appl. Math. Comput., 265 (2015), 1067-1089. 1

[9] A. M. Elaiw, N. A. Almuallem, Global dynamics of delay-distributed HIV infection models with differential drug efficacy in cocirculating target cells, Math. Methods Appl. Sci., 39 (2016), 4-31. 1

[10] A. M. Elaiw, N. H. AlShamrani, Global stability of humoral immunity virus dynamics models with nonlinear infection rate and removal, Nonlinear Anal. Real World Appl., 26 (2015), 161-190. 1

[11] A. M. Elaiw, N. H. AlShamrani, Stability of a general delay-distributed virus dynamics model with multi-staged infected progression and immune response, Math. Methods Appl. Sci., 40 (2017), 699-719.

[12] A. M. Elaiw, S. A. Azoz, Global properties of a class of HIV infection models with Beddington-DeAngelis functional response, Math. Methods Appl. Sci., 36 (2013), 383-394. 1

[13] A. M. Elaiw, I. Hassanien, S. Azoz, Global stability of HIV infection models with intracellular delays, J. Korean Math. Soc., 49 (2012), 779-794. 1

[14] A. M. Elaiw, A. A. Raezah, A. S. Alofi, Effect of humoral immunity on HIV-1 dynamics with virus-to-target and infectedto-target infections, AIP Adv., 6 (2016), 085204. 1

[15] P. Georgescu, Y.-H. Hsieh, Global stability for a virus dynamics model with nonlinear incidence of infection and removal, SIAM J. Appl. Math., 67 (2006), 337-353. 1

[16] K. Hattaf, N. Yousfi, A generalized virus dynamics model with cell-to-cell transmission and cure rate, Adv. Difference Equ., 2016 (2016), 11 pages. 1

[17] G. Huang, W.-B. Ma, Y. Takeuchi, Global analysis for delay virus dynamics model with Beddington-DeAngelis functional response, Appl. Math. Lett., 24 (2011), 1199-1203. 1

[18] G. Huang, Y. Takeuchi, W.-B. Ma, Lyapunov functionals for delay differential equations model of viral infections, SIAM J. Appl. Math., 70 (2010), 2693-2708. 1

[19] D.-W. Huang, X. Zhang, Y.-F. Guo, H.-L. Wang, Analysis of an HIV infection model with treatments and delayed immune response, Appl. Math. Model., 40 (2016), 3081-3089. 1

[20] X.-L. Lai, X.-F. Zou, Modeling HIV-1 virus dynamics with both virus-to-cell infection and cell-to-cell transmission, SIAM J. Appl. Math., 74 (2014), 898-917. 1 
[21] X.-L. Lai, X.-F. Zou, Modeling cell-to-cell spread of HIV-1 with logistic target cell growth, J. Math. Anal. Appl., 426 (2015), 563-584. 1

[22] B. Li, Y.-M. Chen, X.-J. Lu, S.-Q. Liu, A delayed HIV-1 model with virus waning term, Math. Biosci. Eng., 13 (2016), 135-157. 1

[23] M. Y. Li, L.-C. Wang, Backward bifurcation in a mathematical model for HIV infection in vivo with anti-retroviral treatment, Nonlinear Anal. Real World Appl., 17 (2014), 147-160. 1

[24] F. Li, J.-L. Wang, Analysis of an HIV infection model with logistic target-cell growth and cell-to-cell transmission, Chaos Solitons Fractals, 81 (2015), 136-145. 1

[25] S.-Q. Liu, L. Wang, Global stability of an HIV-1 model with distributed intracellular delays and a combination therapy, Math. Biosci. Eng., 7 (2010), 675-685. 1

[26] M. C. Maheswari, P. Krishnapriya, K. Krishnan, M. Pitchaimani, A mathematical model of HIV-1 infection within host cell to cell viral transmissions with RTI and discrete delays, J. Appl. Math. Comput., (2016), 1-28. 1

[27] C. C. McCluskey, Y. Yang, Global stability of a diffusive virus dynamics model with general incidence function and time delay, Nonlinear Anal. Real World Appl., 25 (2015), 64-78. 1

[28] C. Monica, M. Pitchaimani, Analysis of stability and Hopf bifurcation for HIV-1 dynamics with PI and three intracellular delays, Nonlinear Anal. Real World Appl., 27 (2016), 55-69. 1

[29] A. U. Neumann, N. P. Lam, H. Dahari, D. R. Gretch, T. E. Wiley, T. J. Layden, A. S. Perelson, Hepatitis C viral dynamics in vivo and the antiviral efficacy of interferon- $\alpha$ therapy, Science, 282 (1998), 103-107.

[30] M. A. Nowak, C. R. M. Bangham, Population dynamics of immune responses to persistent viruses, Science, 272 (1996), 74-79. 1

[31] P. K. Roy, A. N. Chatterjee, D. Greenhalgh, Q. J. A. Khan, Long term dynamics in a mathematical model of HIV-1 infection with delay in different variants of the basic drug therapy model, Nonlinear Anal. Real World Appl., 14 (2013), $1621-1633$.

[32] X.-G. Shi, X.-Y. Zhou, X.-Y. Son, Dynamical behavior of a delay virus dynamics model with CTL immune response, Nonlinear Anal. Real World Appl., 11 (2010), 1795-1809.

[33] H.-Y. Shu, L. Wang, J. Watmough, Global stability of a nonlinear viral infection model with infinitely distributed intracellular delays and CTL immune responses, SIAM J. Appl. Math., 73 (2013), 1280-1302. 1

[34] X.-Y. Song, A. U. Neumann, Global stability and periodic solution of the viral dynamics, J. Math. Anal. Appl., 329 (2007), 281-297. 1

[35] K. Wang, A.-J. Fan, A. Torres, Global properties of an improved hepatitis B virus model, Nonlinear Anal. Real World Appl., 11 (2010), 3131-3138. 1

[36] J.-L. Wang, M. Guo, X.-N. Liu, Z.-T. Zhao, Threshold dynamics of HIV-1 virus model with cell-to-cell transmission, cell-mediated immune responses and distributed delay, Appl. Math. Comput., 291 (2016), 149-161. 1

[37] L.-C. Wang, M. Y. Li, D. Kirschner, Mathematical analysis of the global dynamics of a model for HTLV-I infection and ATL progression, Math. Biosci., 179 (2002), 207-217. 1

[38] J.-L. Wang, J. Yang, T. Kuniya, Dynamics of a PDE viral infection model incorporating cell-to-cell transmission, J. Math. Anal. Appl., 444 (2016), 1542-1564. 1

[39] S.-H. Xu, Global stability of the virus dynamics model with Crowley-Martin functional response, Electron. J. Qual. Theory Differ. Equ., 2012 (2012), 10 pages. 1

[40] Y. Yang, J.-L. Zhou, X.-S. Ma, T.-H. Zhang, Nonstandard finite difference scheme for a diffusive within-host virus dynamics model with both virus-to-cell and cell-to-cell transmissions, Comput. Math. Appl., 72 (2016), 1013-1020. 1

[41] Y. Yang, L. Zou, S.-G. Ruan, Global dynamics of a delayed within-host viral infection model with both virus-to-cell and cell-to-cell transmissions, Math. Biosci., 270 (2015), 183-191. 1

[42] Y.-Q. Zhao, D. T. Dimitrov, H. Liu, Y. Kuang, Mathematical insights in evaluating state dependent effectiveness of HIV prevention interventions, Bull. Math. Biol., 75 (2013), 649-675. 1 